\title{
GROMOV HYPERBOLICITY OF RIEMANN SURFACES
}

\author{
JOSE M. RODRIGUEZ ${ }^{(1)}$ AND EVA TOURIS ${ }^{(2)}$
}

\begin{abstract}
In this paper we study the hyperbolicity in the Gromov sense of Riemann surfaces. We deduce the hyperbolicity of a surface from the hyperbolicity of its "building block components". We also prove the equivalence between the hyperbolicity of a Riemann surface and the hyperbolicity of some graph associated to it. These results clarify how the decomposition of a Riemann surface in $Y$-pieces and funnels affects on the hyperbolicity of the surface. The results simplify the topology of the surface and allow to obtain global results from local information.
\end{abstract}

\section{INTRODUCTION.}

A good way to understand the important connections between graphs and Potential Theory on Riemannian manifolds (see e.g. [APR], [ARY], [CFPR], [FR2], [HS], [K1], [K2], [K3], [R1], [R2], [So]) is to study Gromov hyperbolic spaces. This approach allows us to establish a general setting to work simultaneously with graphs and manifolds, in the context of metric spaces. Besides, the idea of Gromov hyperbolicity grasps the essence of negatively curved spaces, and has been successfully used in the theory of groups (see e.g. [GH], [G1], [G2] and the references therein).

Although there exist some interesting examples of hyperbolic spaces (see the examples after Definition 2.1), the literature gives no good guide about how to determine whether or not a space is hyperbolic. Recently, some interesting results of Balogh and Buckley [BB] about the hyperbolicity of Euclidean bounded domains with their quasihyperbolic metric have made significant progress in this direction (see also [BHK] and the references therein).

We are interested in studying when non-exceptional Riemann surfaces equipped with their Poincaré metrics are Gromov hyperbolic. We have also proved several theorems on hyperbolicity for general metric spaces, which are interesting by themselves (see Section 2); they are key tools in the study of Riemann surfaces (see Section 3). Although one should expect Gromov hyperbolicity in non-exceptional Riemann surfaces due to its constant curvature -1 , this turns out to be untrue in general, since topological obstacles can impede it: for instance, the two-dimensional jungle-gym (a $\mathbf{Z}^{2}$-covering of a torus with genus two) is not hyperbolic. Let us recall that in the case of modulated plane domains, the quasihyperbolic metric and Poincaré metric are equivalent.

Date: February 27, 2007.

2000 AMS Subject Classification: 30F, 30F20, 30F45.

(1) Research partially supported by a grant from DGI (BFM 2003-04870), Spain.

(2) Research partially supported by a grant from DGI (BFM 2000-0022), Spain. 
We prove in Section 4 that there is no inclusion relationship between hyperbolic Riemann surfaces and the usual classes of Riemann surfaces, such as $O_{G}, O_{H P}, O_{H B}, O_{H D}$, surfaces with hyperbolic isoperimetric inequality, or the complements of these classes (even in the case of plane domains). This fact shows that the study of hyperbolic Riemann surfaces is more complicated and interesting that one might think at first sight. One can find other results on hyperbolicity of Riemann surfaces in [RT1], [RT2] and [PRT2].

Here we present the outline of the main results. We refer to the next sections for the definitions and the precise statements of the theorems.

We can create or delete infinitely many topological obstacles in a metric space, preserving its hyperbolicity (see Theorem 2.2). This fact simplifies the topology of the space (recall that topological obstacles make difficult the hyperbolicity of a space).

One of the important aims in this paper is obtaining global results on hyperbolicity from local information. That was the idea that led us to think of a Riemann surface $S$ as the union of some "pieces" or "building block components" $\left\{S_{n}\right\}$. Theorem 2.1 guarantees the hyperbolicity of some metric spaces which are narrow in some sense. Using this result, we study the role of the decomposition of a Riemann surface in $Y$-pieces and funnels (or more general bordered surfaces) in its hyperbolicity (see theorems 3.1, 3.2, 3.6, 3.7 and 3.8). In particular, theorems 3.2, 3.7 and 3.8 can be applied even in cases with arbitrarily long simple closed geodesics in the boundary of the $Y$-pieces. The hyperbolicity constant in Theorem 2.1 is sharp, and this fact allows us to obtain accurate hyperbolicity constants in Theorem 3.1, and propositions 3.1 and 3.2, and good constants in the other results.

We also have results on uniform hyperbolicity of surfaces of finite type (see theorems 3.4 and 3.5, and propositions 3.1 and 3.2). Theorem 3.5 is remarkable, since it guarantees the hyperbolicity of surfaces of finite type, with hyperbolicity constants which only depend on the topology of the surface and some metric restrictions. By this reason it can be viewed as a result on stability of the hyperbolicity of Riemann surfaces.

Theorem 3.7 is also one of the remarkable results of this paper, since it allows us to simplify significantly the study of the hyperbolicity of a Riemann surface $S$ : it shows how to construct explicitly a very simple graph $G$ related to $S$, such that the hyperbolicity of $G$ guarantees the hyperbolicity of $S$. In theorems 3.1, 3.2 and 3.6 the uniform hyperbolicity of the pieces gives the hyperbolicity of the surface, since the pieces are joined together following a tree-like design (in which no topological obstacles are created). In Theorem 3.7 we cannot obtain the global hyperbolicity just from local information, since we do not have any restriction on the connections of the pieces; it is necessary to ask for the hyperbolicity of the graph used as a model for the connections. This result simplifies the geometry of the surface, since we only need to study its "skeleton".

Theorem 3.7 can be applied to prove that some deformations of Riemann surfaces preserve the hyperbolicity, such as significant changes in the length of simple closed geodesics (see Theorem 3.8) or "twists" in the $Y$-pieces (see Corollary 3.3). 
We want to remark a last result. It is clear that the funnel $F_{l}$ with $L\left(\partial F_{l}\right)=l$ has thin constant $\delta_{l} \geq l / 4$; consequently, one can think that a surface with funnels with arbitrarily long simple closed geodesics cannot be hyperbolic. However, Corollary 3.1 shows that this is not true.

We want to remark that almost every constant appearing in the results of this paper depends just on a small number of parameters. This is a common place in the theory of hyperbolic spaces (see e.g. theorems $\mathrm{A}, \mathrm{B}$ and $\mathrm{C}$ ) and is also typical of surfaces with curvature -1 (see e.g. theorems D and E, the Collar Lemma in $[\mathrm{R}]$ and $[\mathrm{S}]$, and Theorem 3.1 in [PRT2]).

Notations. We denote by $X$ or $X_{n}$ geodesic metric spaces. By $d_{X}, L_{X}$ and $B_{X}$ we shall denote, respectively, the distance, the length and the balls in the metric of $X$.

We denote by $S$ or $S_{n}$ non-exceptional Riemann surfaces. We assume that the metric defined on these surfaces is the Poincaré metric, unless the contrary is specified.

Finally, we denote by $c_{i}, k_{i}$, positive constants which can assume different values in different theorems.

Acknowledgements. We would like to thank Professor José Luis Fernández for some helpful suggestions. Also, we would like to thank Venancio Alvarez, María Auxiliadora Márquez and Ana Portilla for some useful discussions. Also, we would like to thank the referee for his/her careful reading of the manuscript and for some helpful suggestions.

\section{Results in Metric SPACES}

In our study of hyperbolic Gromov spaces we use the notations of [GH]. We give now the basic facts about these spaces. We refer to $[\mathrm{GH}]$ for more background and further results.

Definition 2.1. Let us fix a point $w$ in a metric space $(X, d)$. We define the Gromov product of $x, y \in X$ with respect to the point $w$ as

$$
(x \mid y)_{w}:=\frac{1}{2}(d(x, w)+d(y, w)-d(x, y)) \geq 0 .
$$

We say that the metric space $(X, d)$ is $\delta$-hyperbolic $(\delta \geq 0)$ if

$$
(x \mid z)_{w} \geq \min \left\{(x \mid y)_{w},(y \mid z)_{w}\right\}-\delta,
$$

for every $x, y, z, w \in X$. We say that $X$ is hyperbolic (in the Gromov sense) if the value of $\delta$ is not important.

In this paper we only use the word hyperbolic in the sense of Definition 2.1.

Examples: (1) Every bounded metric space $X$ is (diam $X$ )-hyperbolic (see e.g. [GH, p. 29]).

(2) Every complete simply connected Riemannian manifold with sectional curvature which is bounded from above by $-k$, with $k>0$, is hyperbolic (see e.g. [GH, p. 52]).

(3) Every tree with edges of arbitrary length is 0-hyperbolic (see e.g. [GH, p. 29]). 
Definition 2.2. If $\gamma:[a, b] \longrightarrow X$ is a continuous curve in a metric space $(X, d)$, we can define the length of $\gamma$ as

$$
L(\gamma):=\sup \left\{\sum_{i=1}^{n} d\left(\gamma\left(t_{i-1}\right), \gamma\left(t_{i}\right)\right): a=t_{0}<t_{1}<\cdots<t_{n}=b\right\} .
$$

We say that $\gamma$ is a geodesic if it is an isometry, i.e. $L\left(\left.\gamma\right|_{[t, s]}\right)=d(\gamma(t), \gamma(s))=|t-s|$ for every $s, t \in[a, b]$. We say that $X$ is a geodesic metric space if for every $x, y \in X$ there exists a geodesic joining $x$ and $y$; we denote by $[x, y]$ any of such geodesics (since we do not require uniqueness of geodesics, this notation is ambiguous, but it is convenient). It is clear that every geodesic metric space is path-connected.

Definition 2.3. If $X$ is a geodesic metric space and $x_{1}, x_{2}, x_{3} \in X$, a geodesic triangle $T=\left\{x_{1}, x_{2}, x_{3}\right\}$ is the union of three geodesics $\left[x_{1}, x_{2}\right],\left[x_{2}, x_{3}\right]$ and $\left[x_{3}, x_{1}\right]$. A geodesic triangle $T$ is $\delta$-thin (or satisfies the Rips condition with constant $\delta)$ if for every $x \in\left[x_{i}, x_{j}\right]$ we have that $d\left(x,\left[x_{j}, x_{k}\right] \cup\left[x_{k}, x_{i}\right]\right) \leq \delta$ for any permutation $\left\{x_{i}, x_{j}, x_{k}\right\}$ of $\left\{x_{1}, x_{2}, x_{3}\right\}$. The space $X$ is $\delta$-thin if every geodesic triangle in $X$ is $\delta$-thin.

Remark. If we have a triangle with two identical vertices, we call it a "bigon". Note that since this is a special case of the definition, every bigon in a $\delta$-thin space is $\delta$-thin.

A basic result is that hyperbolicity is equivalent to Rips condition:

Theorem A. ([GH, p. 41]) Let us consider a geodesic metric space $X$.

(1) If $X$ is $\delta$-hyperbolic, then it is $4 \delta$-thin.

(2) If $X$ is $\delta$-thin, then it is $4 \delta$-hyperbolic.

We present now the class of maps which play the main role in the theory.

Definition 2.4. A function between two metric spaces $f: X \longrightarrow Y$ is a quasi-isometry if there are constants $a \geq 1, b \geq 0$ with

$$
\frac{1}{a} d_{X}\left(x_{1}, x_{2}\right)-b \leq d_{Y}\left(f\left(x_{1}\right), f\left(x_{2}\right)\right) \leq a d_{X}\left(x_{1}, x_{2}\right)+b, \quad \text { for every } x_{1}, x_{2} \in X .
$$

Such a function is called an $(a, b)$-quasi-isometry. An $(a, b)$-quasigeodesic in $X$ is an $(a, b)$-quasi-isometry between an interval of $\mathbf{R}$ and $X$. An $(a, b)$-quasigeodesic segment in $X$ is an $(a, b)$-quasi-isometry between a compact interval of $\mathbf{R}$ and $X$.

Notice that a quasi-isometry can be discontinuous.

Quasi-isometries are important since they are maps which preserve hyperbolicity:

Theorem B. ([GH, p. 88]) Let us consider two geodesic metric spaces $X$ and $Y$, and an $(a, b)$-quasiisometry $f$ of $X$ onto $Y$. If $Y$ (respectively $X)$ is $\delta$-hyperbolic, then $X$ (respectively $Y$ ) is $\delta^{\prime}$-hyperbolic, where $\delta^{\prime}$ is a constant which only depends on $\delta, a$ and $b$.

In this paper we will work with topological subspaces of a geodesic metric space $X$. There is a natural way to define a distance in these spaces:

Definition 2.5. If $X_{0}$ is a path-connected subset of a metric space $(X, d)$, then we associate to it the restricted distance

$$
d_{X_{0}}(x, y):=\left.d_{X}\right|_{X_{0}}(x, y):=\inf \left\{L(\gamma): \gamma \subset X_{0} \text { is a continuous curve joining } x \text { and } y\right\} \geq d_{X}(x, y) .
$$


We need an additional definition in order to obtain our first result.

Definition 2.6. A geodesic metric space $X$ is $c_{1}$-decomposible if it verifies:

(1) $X=\cup_{r \in I} X^{r}$, with $I$ an interval in the real line, $\left\{X^{r}\right\}_{r \in I}$ pairwise disjoint, $A(r)$ a set of indices for each $r \in I$ and $X^{r}=\cup_{a \in A(r)} X_{a}^{r}$, with $\left\{X_{a}^{r}\right\}_{a \in A(r)}$ pairwise disjoint closed sets, and $\operatorname{diam}_{X} X_{a}^{r} \leq c_{1}$.

(2) If for each geodesic $\gamma:[0, l] \longrightarrow X$ and $s \in[0, l]$, we denote by $X_{a(s)}^{r(s)}$ the set $X_{a}^{r}$ with $\gamma(s) \in X_{a}^{r}$, then $\cup_{s \in[0, l]} X_{a(s)}^{r(s)}$ is a closed set.

(3) If $X_{a(0)}^{r(0)} \neq X_{a(l)}^{r(l)}$, then there is $s \in(0, l)$ such that $X \backslash X_{a(s)}^{r(s)}$ is not connected, and $x_{0}, x_{l}$ are in different connected components of $X \backslash X_{a(s)}^{r(s)}$, for every $x_{0} \in X_{a(0)}^{r(0)}, x_{l} \in X_{a(l)}^{r(l)}$.

A standard way to obtain a decomposition is to take a continuous function $f: X \longrightarrow \mathbf{R}$, to define $X^{r}:=f^{-1}(\{r\})$ and to consider $\left\{X_{a}^{r}\right\}_{a \in A(r)}$ as the connected components of $X^{r}$. A natural choice of $f$ is $f(x)=d\left(x, x_{0}\right)$, for fixed $x_{0} \in X$. This choice gives the first example of decomposible spaces: the trees are 0-decomposible. Non-trivial examples of decomposible spaces appear in propositions 3.1 and 3.2 , and in theorems $3.1,3.2$ and 3.4 .

Remarks. 1. The item (2) is only a technical topological condition about the "continuity" in $r$ of $X^{r}$, which is trivially satisfied in the applications developed in propositions 3.1 and 3.2, and theorems $3.1,3.2$ and 3.4.

2. If $\gamma:[0, l] \longrightarrow X$ is a geodesic, then $\gamma:[\alpha, \beta] \longrightarrow X$ is also a geodesic for any $0 \leq \alpha<\beta \leq l$. Hence conditions (2) and (3) imply, respectively:

$\left(2^{\prime}\right)$ For each geodesic $\gamma:[0, l] \longrightarrow X$, the set $\cup_{s \in[\alpha, \beta]} X_{a(s)}^{r(s)}$ is closed for any $0 \leq \alpha<\beta \leq l$.

$\left(3^{\prime}\right)$ For any $0 \leq \alpha<\beta \leq l$, if $X_{a(\alpha)}^{r(\alpha)} \neq X_{a(\beta)}^{r(\beta)}$, then there is $s \in(\alpha, \beta)$ such that $X \backslash X_{a(s)}^{r(s)}$ is not connected, and $x_{\alpha}, x_{\beta}$ are in different connected components of $X \backslash X_{a(s)}^{r(s)}$, for every $x_{\alpha} \in X_{a(\alpha)}^{r(\alpha)}, x_{\beta} \in X_{a(\beta)}^{r(\beta)}$.

Theorem 2.1. Every $c_{1}$-decomposible geodesic metric space is $\left(3 c_{1} / 2\right)$-thin.

Proof. The idea of the proof is to show that given a point $x$ in a geodesic triangle $T$, then there exists a set $X_{a}^{r}($ near $x)$ which intersects two sides of $T$.

Let us consider a geodesic triangle $T$ with vertices $\left\{x_{1}, x_{2}, x_{3}\right\}$ and $x \in T$. Without loss of generality we can assume that $x \in\left[x_{1}, x_{2}\right]$. If $l:=d_{X}\left(x_{1}, x_{2}\right)$, we consider the arc-length parametrization of $\left[x_{1}, x_{2}\right]$, $\gamma:[0, l] \longrightarrow X$. Let us denote by $\eta$ the union of the two other sides $\eta:=\left[x_{2}, x_{3}\right] \cup\left[x_{3}, x_{1}\right]$. If $x \in X_{a}^{r}$ and $\eta \cap X_{a}^{r} \neq \varnothing$, then $d_{X}(x, \eta) \leq c_{1}$ by (1), and there is nothing else to prove. Assume that $\eta \cap X_{a}^{r}=\varnothing$; then we will prove $d_{X}(x, \eta) \leq 3 c_{1} / 2$. We consider $s_{1}:=\gamma^{-1}(x) \in(0, l)$. Let us define

$$
\begin{aligned}
& s_{0}:=\inf \left\{\alpha>0: X_{a(s)}^{r(s)} \cap \eta=\varnothing \forall s \in\left(\alpha, s_{1}\right]\right\}, \\
& s_{2}:=\sup \left\{\beta<l: X_{a(s)}^{r(s)} \cap \eta=\varnothing \forall s \in\left[s_{1}, \beta\right)\right\} .
\end{aligned}
$$

We show now that $X_{a\left(s_{0}\right)}^{r\left(s_{0}\right)} \cap \eta \neq \varnothing$ and $X_{a\left(s_{2}\right)}^{r\left(s_{2}\right)} \cap \eta \neq \varnothing$. We only deal with the second case; the first one is similar. By definition of $s_{2}$ we have only two possibilities: $X_{a\left(s_{2}\right)}^{r\left(s_{2}\right)} \cap \eta \neq \varnothing$ or $X_{a\left(t_{k}\right)}^{r\left(t_{k}\right)} \cap \eta \neq \varnothing$ with $t_{k} \searrow s_{2}$. Let us assume that we have the second possibility; then we can choose $x_{k} \in X_{a\left(t_{k}\right)}^{r\left(t_{k}\right)} \cap \eta$. Since $\eta$ is a compact set, we can choose a subsequence (which we also denote by $x_{k}$ ) and a point $x_{0} \in \eta$ with $x_{k} \rightarrow x_{0}$. For each $\varepsilon>0$ 
there exists $N$ such that $x_{k} \in \eta \cap\left(\cup_{s \in\left[s_{2}, s_{2}+\varepsilon\right]} X_{a(s)}^{r(s)}\right)$, for every $k \geq N$. Recall that $\eta \cap\left(\cup_{s \in\left[s_{2}, s_{2}+\varepsilon\right]} X_{a(s)}^{r(s)}\right)$ is a closed set by $\left(2^{\prime}\right)$. Then $x_{0} \in \eta \cap\left(\cup_{s \in\left[s_{2}, s_{2}+\varepsilon\right]} X_{a(s)}^{r(s)}\right)$, for every $\varepsilon>0$.

First of all we will prove

$$
\bigcap_{\varepsilon>0} \cup_{s \in\left[s_{2}, s_{2}+\varepsilon\right]} X_{a(s)}^{r(s)}=X_{a\left(s_{2}\right)}^{r\left(s_{2}\right)} .
$$

It is clear that $X_{a\left(s_{2}\right)}^{r\left(s_{2}\right)} \subseteq \cap_{\varepsilon>0} \cup_{s \in\left[s_{2}, s_{2}+\varepsilon\right]} X_{a(s)}^{r(s)}$. In order to check the other inclusion let us consider any $y \in \cap_{\varepsilon>0} \cup_{s \in\left[s_{2}, s_{2}+\varepsilon\right]} X_{a(s)}^{r(s)}$. Since $y$ belongs to this intersection, there exists a non-increasing sequence $\left\{u_{n}\right\}_{n}$ converging to $s_{2}$, such that $y \in X_{a\left(u_{n}\right)}^{r\left(u_{n}\right)}$ for every $n$, and then $X_{a\left(u_{n}\right)}^{r\left(u_{n}\right)}=X_{a\left(u_{1}\right)}^{r\left(u_{1}\right)}$ for every $n$.

Since $X_{a\left(u_{1}\right)}^{r\left(u_{1}\right)}$ is a closed set, $\lim _{n \rightarrow \infty} \gamma\left(u_{n}\right)=\gamma\left(s_{2}\right)$ and $\gamma\left(u_{n}\right) \in X_{a\left(u_{1}\right)}^{r\left(u_{1}\right)}$, then $\gamma\left(s_{2}\right) \in X_{a\left(u_{1}\right)}^{r\left(u_{1}\right)}$; therefore $X_{a\left(u_{1}\right)}^{r\left(u_{1}\right)}=X_{a\left(s_{2}\right)}^{r\left(s_{2}\right)}$, since $\gamma\left(s_{2}\right) \in X_{a\left(s_{2}\right)}^{r\left(s_{2}\right)}$ by definition. Consequently, $y \in X_{a\left(s_{2}\right)}^{r\left(s_{2}\right)}$ as we want to check.

Following with the proof of the theorem, we can conclude that $x_{0} \in \eta \cap X_{a\left(s_{2}\right)}^{r\left(s_{2}\right)}$; hence, $X_{a\left(s_{2}\right)}^{r\left(s_{2}\right)} \cap \eta \neq \varnothing$.

We prove now that $X_{a\left(s_{0}\right)}^{r\left(s_{0}\right)}=X_{a\left(s_{2}\right)}^{r\left(s_{2}\right)}$. Seeking a contradiction, let us assume that this is not true. By $\left(3^{\prime}\right)$ we can take $s \in\left(s_{0}, s_{2}\right)$ such that $X \backslash X_{a(s)}^{r(s)}$ is not connected, and $\gamma\left(s_{0}\right), \gamma\left(s_{2}\right)$ are in different connected components of $X \backslash X_{a(s)}^{r(s)}$; also by $\left(3^{\prime}\right)$, the same is true if we change $\gamma\left(s_{0}\right)$ by any point in $X_{a\left(s_{0}\right)}^{r\left(s_{0}\right)}$ and $\gamma\left(s_{2}\right)$ by any point in $X_{a\left(s_{2}\right)}^{r\left(s_{2}\right)}$.

Consider now a parametrization of the curve $\eta:\left[0, l_{3}\right] \longrightarrow X$. Since $X_{a\left(s_{0}\right)}^{r\left(s_{0}\right)} \cap \eta \neq \varnothing$ and $X_{a\left(s_{2}\right)}^{r\left(s_{2}\right)} \cap \eta \neq \varnothing$, we can choose $0 \leq l_{1}<l_{2} \leq l_{3}$ with $\eta\left(l_{1}\right) \in X_{a\left(s_{0}\right)}^{r\left(s_{0}\right)}, \eta\left(l_{2}\right) \in X_{a\left(s_{2}\right)}^{r\left(s_{2}\right)}$ (or viceverse). We denote by $\eta_{0}:\left[l_{1}, l_{2}\right] \longrightarrow X$ the restriction of $\eta$ to $\left[l_{1}, l_{2}\right]$. It is clear that $\eta_{0} \cap X_{a(s)}^{r(s)}=\varnothing$ (since $\left.\eta \cap X_{a(s)}^{r(s)}=\varnothing\right)$ and therefore, $\eta_{0}$ joins $\eta\left(l_{1}\right) \in X_{a\left(s_{0}\right)}^{r\left(s_{0}\right)}$ with $\eta\left(l_{2}\right) \in X_{a\left(s_{2}\right)}^{r\left(s_{2}\right)}$ in $X \backslash X_{a(s)}^{r(s)}$, which is a contradiction. Therefore $X_{a\left(s_{0}\right)}^{r\left(s_{0}\right)}=X_{a\left(s_{2}\right)}^{r\left(s_{2}\right)}$.

Since $X_{a\left(s_{0}\right)}^{r\left(s_{0}\right)}=X_{a\left(s_{2}\right)}^{r\left(s_{2}\right)}$, we have that $L_{X}\left(\gamma\left(\left[s_{0}, s_{2}\right]\right)\right)=d_{X}\left(\gamma\left(s_{0}\right), \gamma\left(s_{2}\right)\right) \leq \operatorname{diam}_{X}\left(X_{a\left(s_{0}\right)}^{r\left(s_{0}\right)}\right) \leq c_{1}$. Recall that $x=\gamma\left(s_{1}\right)$. Consequently $d_{X}\left(x, X_{a\left(s_{0}\right)}^{r\left(s_{0}\right)}\right) \leq \min \left\{L_{X}\left(\gamma\left(\left[s_{0}, s_{1}\right]\right)\right), L_{X}\left(\gamma\left(\left[s_{1}, s_{2}\right]\right)\right)\right\} \leq c_{1} / 2$, and so $d_{X}(x, \eta) \leq d_{X}\left(x, X_{a\left(s_{0}\right)}^{r\left(s_{0}\right)}\right)+\operatorname{diam}_{X}\left(X_{a\left(s_{0}\right)}^{r\left(s_{0}\right)}\right) \leq c_{1} / 2+c_{1}=3 c_{1} / 2$.

Definition 2.7. Let us consider a geodesic metric space $X$ and $\left\{\eta_{n}^{1}, \eta_{n}^{2}\right\}_{n}$ pairwise disjoint compact subsets of $X$. If $c_{1}, c_{2}, c_{3}, c_{4}$ are positive constants, we say that $\left\{\eta_{n}^{1}, \eta_{n}^{2}\right\}_{n}$ are $\left(c_{1}, c_{2}, c_{3}, c_{4}\right)$-identified if:

(1) there exists a bijective isometry $f_{n}:\left(\eta_{n}^{1},\left.d_{X}\right|_{\eta_{n}^{1}}\right) \longrightarrow\left(\eta_{n}^{2},\left.d_{X}\right|_{\eta_{n}^{2}}\right)$ for each $n$,

(2) $d_{X}\left(p, p^{\prime}\right) \leq c_{1}$ if $f_{n}(p)=p^{\prime}$ for some $n$,

(3) $d_{X}\left(\eta_{n}^{1} \cup \eta_{n}^{2}, \eta_{m}^{1} \cup \eta_{m}^{2}\right) \geq c_{2}$ for every $n \neq m$,

(4) if we denote by $X_{0}$ the space obtained by identifying in $X$ the closed sets $\eta_{n}^{1}$ and $\eta_{n}^{2}$ by $f_{n}$ for each $n$, and by $f$ the canonical projection of $X$ onto $X_{0}$, then for each $n$ there exists $i \in\{1,2\}$ with $d_{X}(u, v) \leq c_{3} d_{X_{0}}(f(u), f(v))+c_{4}$ if $u, v \in \eta_{n}^{i}$.

Remarks. 1. Hypothesis (3) guarantees that $d_{X_{0}}$ (defined by Definition 2.5) is a distance.

2. Conditions (2) and (4) are satisfied if $\operatorname{diam}_{X}\left(\eta_{n}^{1} \cup \eta_{n}^{2}\right) \leq c$ for every $n$.

The following theorem allows us to create infinitely many topological obstacles in a metric space ("genus", if the space is a surface), preserving its hyperbolicity. 
There is a more useful point of view to appreciate the next theorem: we can delete infinitely many topological obstacles in a metric space, preserving its hyperbolicity. This fact allows a great simplification in the topology of the space (recall that topological obstacles make difficult the hyperbolicity).

Theorem 2.2. Let us consider a geodesic metric space $X$ and $\left\{\eta_{n}^{1}, \eta_{n}^{2}\right\}_{n}\left(c_{1}, c_{2}, c_{3}, c_{4}\right)$-identified. Then the canonical projection $f$ of $X$ onto $X_{0}$ is a quasi-isometry with constants which only depend on $c_{1}, c_{2}, c_{3}, c_{4}$. Consequently, if $X_{0}$ is a geodesic metric space, then $X$ is hyperbolic if and only if $X_{0}$ is hyperbolic. In particular, if $X$ (respectively $\left.X_{0}\right)$ is $\delta$-hyperbolic, then $X_{0}$ (respectively $\left.X\right)$ is $\delta^{\prime}$-hyperbolic, with $\delta^{\prime}$ a universal constant which only depends on $\delta, c_{1}, c_{2}, c_{3}$ and $c_{4}$.

Remarks. 1. It is possible to prove (we can apply a similar argument to the one in the proof of [RT1, Theorem 2.1]) that $X_{0}$ is a geodesic metric space if each ball in $X$ intersects only a finite number of $\eta_{n}^{i}$ 's (this is the case if $X$ is proper).

2. If $\eta_{n}^{i}$ are simple closed curves, the condition that $\left(\eta_{n}^{1},\left.d_{X}\right|_{\eta_{n}^{1}}\right)$ and $\left(\eta_{n}^{2},\left.d_{X}\right|_{\eta_{n}^{2}}\right)$ are isometric is equivalent to $L_{X}\left(\eta_{n}^{1}\right)=L_{X}\left(\eta_{n}^{2}\right)$.

3. Theorem 2.2 is an improvement of [RT1, Theorem 2.2]; furthermore, it uses simpler and shorter arguments.

Proof. We have that $\left.d_{X}\right|_{\eta_{n}^{1}},\left.d_{X}\right|_{\eta_{n}^{2}}$ and $d_{X_{0}}$ (defined by Definition 2.5) are distances.

It is clear that for every curve $\sigma$ in $X$ we have $L_{X}(\sigma)=L_{X_{0}}(f(\sigma))$. Then for every $x, y \in X$ we have $d_{X_{0}}(f(x), f(y)) \leq d_{X}(x, y)$, since there are more curves joining $f(x)$ and $f(y)$ in $X_{0}$ than curves joining $x$ and $y$ in $X$.

In order to prove the other inequality, let us fix $x, y \in X$ and let us consider a geodesic $\gamma_{0}:[0, l] \longrightarrow X_{0}$ joining $f(x)$ and $f(y)$, if there exists such geodesic (if this was not so, we can take $\gamma_{k}$ with $L_{X_{0}}\left(\gamma_{k}\right) \leq$ $\left.d_{X_{0}}(f(x), f(y))+1 / k\right)$. Let us define $\eta_{n}:=f\left(\eta_{n}^{1}\right)=f\left(\eta_{n}^{2}\right)$. Then $d_{X_{0}}\left(\eta_{n}, \eta_{m}\right) \geq c_{2}$ if $n \neq m$, by (3).

If $L_{X_{0}}\left(\gamma_{0}\right)=d_{X}(x, y)$, then $d_{X_{0}}(f(x), f(y))=d_{X}(x, y)$ and we are done. So suppose this is not so. We shall construct a continuous curve $g$ in $X$ joining $x$ and $y$, related to $\gamma_{0}$. If $L_{X_{0}}\left(\gamma_{0}\right)<d_{X}(x, y)$, then $\gamma_{0}$ meets some $\eta_{n}$. In this case let us choose a finite union of curves $\gamma$ in $X$ as follows: Since $d_{X_{0}}\left(\eta_{n}, \eta_{m}\right) \geq c_{2}$, $\gamma_{0}$ intersects only a finite number of $\eta_{n}$ 's. Let us define

$$
t_{1}^{1}:=\min \left\{0 \leq t \leq l: \gamma_{0}(t) \in \cup_{n} \eta_{n}\right\} .
$$

There exists this minimum since $\gamma_{0}$ is a continuous function in a compact interval and $\gamma_{0} \cap\left(\cup_{n} \eta_{n}\right)$ is a compact set ( $\gamma_{0}$ intersects only a finite number of $\eta_{n}$ 's).

Then there is $n_{1}$ such that $\gamma_{0}\left(t_{1}^{1}\right) \in \eta_{n_{1}}$, and we define

$$
t_{1}^{2}:=\max \left\{0 \leq t \leq l: \gamma_{0}(t) \in \eta_{n_{1}}\right\} .
$$

In a similar way, we define recursively

$$
t_{j}^{1}:=\min \left\{t_{j-1}^{2}<t \leq l: \gamma_{0}(t) \in \cup_{n} \eta_{n}\right\} ;
$$

if $\gamma_{0}\left(t_{j}^{1}\right) \in \eta_{n_{j}}$, for some $n_{j}$, we take

$$
t_{j}^{2}:=\max \left\{t_{j-1}^{2}<t \leq l: \gamma_{0}(t) \in \eta_{n_{j}}\right\} .
$$


We can continue this choice for $1 \leq j \leq r$. We define a finite union of curves $\gamma$ in $X$ as the restriction of $f^{-1}\left(\gamma_{0}\right)$ to the closed set $\left[0, t_{1}^{1}\right] \cup\left[t_{1}^{2}, t_{2}^{1}\right] \cup \cdots \cup\left[t_{r-1}^{2}, t_{r}^{1}\right] \cup\left[t_{r}^{2}, l\right]$; since $f$ is not injective, we take $\gamma\left(t_{j}^{1}\right):=$ $\lim _{t \rightarrow\left(t_{j}^{1}\right)-\gamma(t)}$ and $\gamma\left(t_{j}^{2}\right):=\lim _{t \rightarrow\left(t_{j}^{2}\right)^{+}} \gamma(t)$; if $t_{1}^{1}=0$ (and/or $\left.t_{r}^{2}=l\right)$, we take $\gamma(0)=x$ (and/or $\left.\gamma(l)=y\right)$.

Notice that $\gamma\left(t_{j}^{1}\right) \in \eta_{n_{j}}^{i}, \gamma\left(t_{j}^{2}\right) \in \eta_{n_{j}}^{k}$, with $i, k \in\{1,2\}$, and $i$ can be equal or not to $k$.

Now, let us choose continuous curves $g_{j}$ connecting $\gamma\left(t_{j}^{1}\right)$ and $\gamma\left(t_{j}^{2}\right)$ in $X$ in the following way: by (4), we can take $i \in\{1,2\}$ and a geodesic $h_{j}$ in $X$ joining $\gamma\left(t_{j}^{1}\right)^{*}$ and $\gamma\left(t_{j}^{2}\right)^{*}$, with $L_{X}\left(h_{j}\right)=d_{X}\left(\gamma\left(t_{j}^{1}\right)^{*}, \gamma\left(t_{j}^{2}\right)^{*}\right) \leq$ $c_{3} d_{X_{0}}\left(f\left(\gamma\left(t_{j}^{1}\right)\right), f\left(\gamma\left(t_{j}^{2}\right)\right)\right)+c_{4}$, where $\gamma\left(t_{j}^{k}\right)^{*} \in \eta_{n_{j}}^{i} \cap\left\{f^{-1}\left(\gamma_{0}\left(t_{j}^{k}\right)\right)\right\}$ for $k=1,2$. Then $g_{j}$ is the union of $h_{j}$ and at most two curves of length less or equal than $c_{1}$, by (2); therefore $L_{X}\left(g_{j}\right) \leq c_{3} d_{X_{0}}\left(\gamma_{0}\left(t_{j}^{1}\right), \gamma_{0}\left(t_{j}^{2}\right)\right)+2 c_{1}+c_{4}$.

We define $g:=\gamma \cup g_{1} \cup g_{2} \cup \cdots \cup g_{r}$, which is a continuous curve in $X$ joining $x$ and $y$. Consequently we have

$$
\begin{aligned}
d_{X}(x, y) \leq & L_{X}(g)=L_{X}(\gamma)+\sum_{j=1}^{r} L_{X}\left(g_{j}\right) \\
\leq & d_{X_{0}}\left(\gamma_{0}(0), \gamma_{0}\left(t_{1}^{1}\right)\right)+\sum_{j=1}^{r-1} d_{X_{0}}\left(\gamma_{0}\left(t_{j}^{2}\right), \gamma_{0}\left(t_{j+1}^{1}\right)\right)+d_{X_{0}}\left(\gamma_{0}\left(t_{r}^{2}\right), \gamma_{0}(l)\right) \\
& +\sum_{j=1}^{r}\left(c_{3} d_{X_{0}}\left(\gamma_{0}\left(t_{j}^{1}\right), \gamma_{0}\left(t_{j}^{2}\right)\right)+2 c_{1}+c_{4}\right)
\end{aligned}
$$

Notice that

$$
(r-1)\left(2 c_{1}+c_{4}\right) \leq \frac{2 c_{1}+c_{4}}{c_{2}} \sum_{j=1}^{r-1} d_{X_{0}}\left(\gamma_{0}\left(t_{j}^{2}\right), \gamma_{0}\left(t_{j+1}^{1}\right)\right),
$$

since $d_{X_{0}}\left(\eta_{n}, \eta_{m}\right) \geq c_{2}$. Then

$$
\begin{aligned}
d_{X}(x, y) \leq & d_{X_{0}}\left(\gamma_{0}(0), \gamma_{0}\left(t_{1}^{1}\right)\right)+\left(1+\frac{2 c_{1}+c_{4}}{c_{2}}\right) \sum_{j=1}^{r-1} d_{X_{0}}\left(\gamma_{0}\left(t_{j}^{2}\right), \gamma_{0}\left(t_{j+1}^{1}\right)\right) \\
& +d_{X_{0}}\left(\gamma_{0}\left(t_{r}^{2}\right), \gamma_{0}(l)\right)+c_{3} \sum_{j=1}^{r} d_{X_{0}}\left(\gamma_{0}\left(t_{j}^{1}\right), \gamma_{0}\left(t_{j}^{2}\right)\right)+2 c_{1}+c_{4} \\
\leq & c d_{X_{0}}(f(x), f(y))+2 c_{1}+c_{4},
\end{aligned}
$$

where $c:=\max \left\{1+\left(2 c_{1}+c_{4}\right) / c_{2}, c_{3}\right\}$. We conclude that $f$ is a quasi-isometry with constants which only depend on $c_{1}, c_{2}, c_{3}, c_{4}$. The conclusions about hyperbolicity are a consequence of this fact and Theorem B.

We finish this section with a theorem which will be very useful in the proof of the main results of this paper. In order to state it, we need a definition.

Definition 2.8. We say that a geodesic metric space $X$ has a decomposition, if there exists a family of geodesic metric spaces $\left\{X_{n}\right\}_{n \in \Lambda}$ with $X=\cup_{n \in \Lambda} X_{n}$ and $X_{n} \cap X_{m}=\eta_{n m}$, where for each $n \in \Lambda,\left\{\eta_{n m}\right\}_{m}$ are pairwise disjoint closed subsets of $X_{n}\left(\eta_{n m}=\varnothing\right.$ is allowed); furthermore any geodesic segment in $X$ meets at most a finite number of $\eta_{n m}$ 's.

We say that $X_{n}$, with $n \in \Lambda$, is a $\left(k_{1}, k_{2}, k_{3}\right)$-tree-piece if it satisfies the following properties: 
(a) If $\eta_{n m} \neq \varnothing$, then $X \backslash \eta_{n m}$ is not connected and $a, b$ are in different connected components of $X \backslash \eta_{n m}$ for any $a \in X_{n} \backslash \eta_{n m}, b \in X_{m} \backslash \eta_{n m}$.

(b) $\operatorname{diam}_{X_{n}}\left(\eta_{n m}\right) \leq k_{1}$ for every $m \neq n$, and there exists $A_{n} \subseteq \Lambda$, such that $\operatorname{diam}_{X_{n}}\left(\eta_{n m}\right) \leq k_{2} d_{X_{n}}\left(\eta_{n m}, \eta_{n k}\right)$ if $m \neq k$ and $m, k \in A_{n}$, and $\sum_{m \notin A_{n}} \operatorname{diam}_{X_{n}}\left(\eta_{n m}\right) \leq k_{3}$.

We say that a geodesic metric space $X$ has a $\left(k_{1}, k_{2}, k_{3}\right)$-tree-decomposition if it has a decomposition such that every $X_{n}$, with $n \in \Lambda$, is a $\left(k_{1}, k_{2}, k_{3}\right)$-tree-piece.

We wish to emphasize that condition $\operatorname{diam}_{X_{n}}\left(\eta_{n m}\right) \leq k_{1}$ is not very restrictive: if the space is "wide" at every point (in the sense of long injectivity radius, as in the case of simply connected spaces) or "narrow" at every point (as in the case of trees), it is easier to study its hyperbolicity; if we can find narrow parts (as $\left.\eta_{n m}\right)$ and wide parts, the problem is more difficult and interesting.

Remarks. 1. Obviously, condition (b) is required only for $\eta_{n m}, \eta_{n k} \neq \varnothing$.

2. The sets $\Lambda$ and $A_{n}$ do not need to be countable.

3. The hypothesis $\operatorname{diam}_{X_{n}}\left(\eta_{n m}\right) \leq k_{2} d_{X_{n}}\left(\eta_{n m}, \eta_{n k}\right)$ holds if we have $d_{X_{n}}\left(\eta_{n m}, \eta_{n k}\right) \geq k_{2}^{\prime}$, since $\operatorname{diam}_{X_{n}}\left(\eta_{n m}\right) \leq$ $k_{1}$.

4. Condition (a) for every $n \in \Lambda$ guarantees that the graph $R=(V, E)$ constructed in the following way is a tree: $V=\cup_{n \in \Lambda}\left\{v_{n}\right\}$ and $\left[v_{n}, v_{m}\right] \in E$ if and only if $\eta_{n m} \neq \varnothing$.

5. If $X$ is a Riemann surface, $\left\{X_{n}\right\}_{n \in \Lambda}$ are bordered Riemann surfaces and $\eta_{n m} \subset \partial X_{n} \cap \partial X_{m}$, then the condition " $a, b$ are in different components of $X \backslash \eta_{n m}$ for any $a \in X_{n} \backslash \eta_{n m}, b \in X_{m} \backslash \eta_{n m}$ " in ( $a$ ), is a consequence of " $X \backslash \eta_{n m}$ is not connected".

The following result can be applied to the study of the hyperbolicity of Riemann surfaces (see theorems 3.4, 3.6 and 3.8). In [PRT1] explicit expressions for the constants involved are supplied.

Theorem C. ([PRT1, Theorem 1]) Let us consider a $\left(k_{1}, k_{2}, k_{3}\right)$-tree-decomposition $\left\{X_{n}\right\}_{n \in \Lambda}$ of a geodesic metric space $X$. Then $X$ is $\delta$-hyperbolic if and only if there exists a constant $k_{4}$ such that $X_{n}$ is $k_{4}$-hyperbolic for every $n \in \Lambda$. Furthermore, if $X$ is $\delta$-hyperbolic, then $k_{4}$ only depends on $\delta, k_{1}, k_{2}$ and $k_{3}$; if there exists $k_{4}$, then $\delta$ only depends on $k_{1}, k_{2}, k_{3}$ and $k_{4}$.

\section{Results in Riemann surfaces}

In this section we always work with the Poincaré metric; consequently, curvature is always -1 . In fact, many concepts appearing here (as punctures or funnels) only make sense with the Poincaré metric.

The intuition would say that negative curvature must imply hyperbolicity; in fact this is what happens when there are no topological "obstacles" (as in the case of the Poincaré disk $\mathbf{D}$ ) or if there is a finite number of them (see Theorem 3.5). However, if there are infinitely many topological "obstacles", the hyperbolicity can fail, as in the case of the two-dimensional jungle gym (a $\mathbf{Z}^{2}$-covering of a torus with genus two).

The results in this section are useful since they not only provide many examples of hyperbolic Riemann surfaces, but also allow to establish criteria in order to decide whether a Riemann surface is hyperbolic or not. 
Below we collect some definitions concerning to Riemann surfaces which will be referred to afterwards.

An open non-exceptional Riemann surface (or a non-exceptional Riemann surface without boundary) $S$ is a Riemann surface whose universal covering space is the unit disk $\mathbf{D}=\{z \in \mathbf{C}:|z|<1\}$, endowed with its Poincaré metric, i.e. the metric obtained by projecting the Poincaré metric of the unit disk $d s=2|d z| /(1-$ $|z|^{2}$ ), or, equivalently, the upper half plane $\mathbf{U}=\{z \in \mathbf{C}: \operatorname{Im} z>0\}$, with the metric $d s=|d z| / \operatorname{Im} z$. Notice that, with this definition, every compact non-exceptional Riemann surface without boundary is open. With this metric, $S$ is a geodesically complete Riemannian manifold with constant curvature -1 , and therefore $S$ is a geodesic metric space. The only Riemann surfaces which are left out are the sphere, the plane, the punctured plane and the tori. It is easy to study the hyperbolicity of these particular cases.

Let $S$ be an open non-exceptional Riemann surface with a puncture or cusp $q$ (if $S \subset \mathbf{C}$, every isolated point in $\partial S$ is a puncture). A collar in $S$ about $q$ is a doubly connected domain in $S$ "bounded" both by $q$ and a Jordan curve (called the boundary curve of the collar) orthogonal to the pencil of geodesics emanating from $q$.

A collar in $S$ about $q$ of area $\alpha$ will be called an $\alpha$-collar and it will be denoted by $C_{S}(q, \alpha)$. A theorem of Shimizu [S] gives that for every puncture in any open non-exceptional Riemann surface, there exists an $\alpha$-collar for every $0<\alpha \leq 2$ (see also [Bu, Chapter 4.4]).

We say that a curve is homotopic to a puncture $q$ if it is freely homotopic to $\partial C_{S}(q, \alpha)$ for some (and then for every) $0<\alpha<2$.

We have used the word geodesic in the sense of Definition 2.2, that is to say, as a global geodesic or a minimizing geodesic; however, we need now to deal with a special type of local geodesics: simple closed geodesics, which obviously cannot be minimizing geodesics. We will continue using the word geodesic with the meaning of Definition 2.2, unless we are dealing with closed geodesics.

A collar in $S$ about a simple closed geodesic $\gamma$ is a doubly connected domain in $S$ "bounded" by two Jordan curves (called the boundary curves of the collar) orthogonal to the pencil of geodesics emanating from $\gamma$; such collar is equal to $\left\{p \in S: d_{S}(p, \gamma)<d\right\}$, for some positive constant $d$. The constant $d$ is called the width of the collar. The Collar Lemma $[\mathrm{R}]$ says that there exists a collar of $\gamma$ of width $d$, for every $0<d \leq d_{0}$, where $\cosh d_{0}=\operatorname{coth}\left(L_{S}(\gamma) / 2\right)$.

We say that $S$ is a bordered non-exceptional Riemann surface (or a non-exceptional Riemann surface with boundary) if it can be obtained by deleting an open set $V$ of an open non-exceptional Riemann surface $R$, such that:

(1) $S$ is connected and $d_{S}:=\left.d_{R}\right|_{S}$ (recall Definition 2.5),

(2) any ball in $R$ intersects at most a finite number of connected components of $V$,

(3) the boundary of $S$ is locally Lipschitz.

Any such surface $S$ is a bordered orientable Riemannian manifold of dimension 2 and its Riemannian metric has constant negative curvature -1 . It is not difficult to see that $S$ is a geodesic metric space. 
A funnel is a bordered non-exceptional Riemann surface which is topologically a cylinder and whose boundary is a simple closed geodesic. Given a positive number $a$, there is a unique (up to conformal mapping) funnel such that its boundary curve has length $a$. Every funnel is conformally equivalent, for some $\beta>1$, to the subset $\{z \in \mathbf{C}: 1 \leq|z|<\beta\}$ of the annulus $\{z \in \mathbf{C}: 1 / \beta<|z|<\beta\}$.

Every doubly connected end of an open non-exceptional Riemann surface is a puncture (if there are homotopically non-trivial curves with arbitrary small length) or a funnel (if this was not so).

A $Y$-piece is a bordered non-exceptional Riemann surface which is conformally equivalent to a sphere without three open disks and whose boundary curves are simple closed geodesics. Given three positive numbers $a, b, c$, there is a unique (up to conformal mapping) $Y$-piece such that their boundary curves have lengths $a, b, c$ (see e.g. [Bu, p. 109]). They are a standard tool for constructing Riemann surfaces. A clear description of these $Y$-pieces and their use is given in [C, Chapter X.3] and [Bu, Chapter 3].

A generalized Y-piece is a non-exceptional Riemann surface (with or without boundary) which is conformally equivalent to a sphere without $n$ open disks and $m$ points, with integers $n, m \geq 0$ such that $n+m=3$, the $n$ boundary curves are simple closed geodesics and the $m$ deleted points are punctures. Notice that a generalized $Y$-piece is topologically the union of a $Y$-piece and $m$ cylinders, with $0 \leq m \leq 3$.

We deduce now several applications of Theorem 2.1 which guarantee the hyperbolicity of many Riemann surfaces, with good control of the hyperbolicity constants.

Proposition 3.1. Any generalized $Y$-piece $Y_{0}$ with $L(\gamma) \leq l$, for every simple closed geodesic $\gamma \subseteq \partial Y_{0}$, is $\left(4 r_{0}+l\right)$-decomposible and $3\left(4 r_{0}+l\right) / 2$-thin, where $r_{0}:=2 \operatorname{Arcsinh}(1 / 2)$.

Proof. Let us denote by $\gamma_{1}, \gamma_{2}, \gamma_{3}$, the simple closed geodesics in $\partial Y_{0}$ (as usual, we identify a puncture with a geodesic of zero length). If $\{i, j, k\}$ is any permutation of $\{1,2,3\}$, let us consider the geodesic $B_{i}$ in $Y_{0}$ which is orthogonal to $\gamma_{j}$ and $\gamma_{k}$. If we split $Y_{0}$ along the curves $B_{i}$, we obtain two isometric convex rightangle hexagons $H_{1}, H_{2}$, with consecutive sides of length $L\left(\gamma_{1}\right) / 2, L\left(B_{3}\right), L\left(\gamma_{2}\right) / 2, L\left(B_{1}\right), L\left(\gamma_{3}\right) / 2, L\left(B_{2}\right)$.

Let us consider the middle point $x_{i}$ of the side with length $L\left(\gamma_{i}\right) / 2$ in $H_{1}$, and the geodesic triangle $T=\left\{x_{1}, x_{2}, x_{3}\right\} \subset H_{1}$. We can draw a ball $B\left(z_{0}, r\right)$ contained in $H_{1}$, which is tangent to some $y_{1} \in\left[x_{2}, x_{3}\right]$, $y_{2} \in\left[x_{1}, x_{3}\right]$ and $y_{3} \in\left[x_{1}, x_{2}\right]$. We have that $\pi \geq A(T)>A\left(B\left(z_{0}, r\right)\right)=4 \pi \sinh ^{2}(r / 2)$, and then $r<r_{0}$.

Let us consider the geodesics $\alpha_{1}, \alpha_{2}, \alpha_{3}$ in $H_{1}$ starting in $z_{0}$ and finishing respectively in $x_{1}, x_{2}, x_{3}$.

For each point $p \in\left[x_{i}, x_{j}\right] \subset T$, we consider the geodesic $a_{p}$ in $H_{1}$ which starts in $p$ orthogonally to $\left[x_{i}, x_{j}\right]$ and finishes in $\alpha_{i} \cup \alpha_{j}$, and the geodesic $b_{p}$ in $H_{1}$ which starts orthogonally to $B_{k}$ and finishes in $p$. It is clear that $L\left(a_{p}\right)+L\left(b_{p}\right)<r_{0}+l / 4$.

Therefore we can draw in $H_{1}$ curves joining two of the sets $B_{1}, B_{2}, B_{3}$, with diameter less than $2 r_{0}+l / 2$; in fact, the "curve" containing $z_{0}$ is the union of three curves and joins the three sets.

We can do the same design in $H_{2}$, since it is isometric to $H_{1}$. If we paste these hexagons, we have that $Y_{0}$ is $\left(4 r_{0}+l\right)$-decomposible. Theorem 2.1 gives that $Y_{0}$ is $3\left(4 r_{0}+l\right) / 2$-thin.

Many Riemann surfaces can be decomposed in a union of funnels and generalized $Y$-pieces (see [FM, Theorem 4.1] and $[\mathrm{AR}]$ ). The following results use this decomposition in order to obtain hyperbolicity. 
Theorem 3.1. Let us consider a non-exceptional Riemann surface $S$ (with or without boundary) without genus ( $S$ can be viewed as a plane domain). If there is a decomposition of $S$ in a union of generalized $Y$ pieces $\left\{Y_{n}\right\}_{n \in N}$ with $L_{S}(\gamma) \leq l$ for every simple closed geodesic $\gamma \subset \cup_{n} \partial Y_{n}$, then $S$ is $\left(4 r_{0}+l\right)$-decomposible and $3\left(4 r_{0}+l\right) / 2$-thin, where $r_{0}:=2 \operatorname{Arcsinh}(1 / 2)$.

Proof. By Proposition 3.1 we know that each $Y_{n}$ is $\left(4 r_{0}+l\right)$-decomposible. Since $S$ is a plane domain, the union in $n$ of the curves constructed in Proposition 3.1 in each $Y_{n}$ gives that $S$ is also $\left(4 r_{0}+l\right)$-decomposible, since any of such curves disconnects $S$. Consequently, Theorem 2.1 gives that $S$ is $3\left(4 r_{0}+l\right) / 2$-thin.

With an additional idea we can improve Proposition 3.1 and Theorem 3.1.

Proposition 3.2. Any generalized $Y$-piece $Y_{0}$ with $L(\gamma) \leq l$, for at least two simple closed geodesics $\gamma \subseteq \partial Y_{0}$, is $\left(2 r_{1}+l\right)$-decomposible and $3\left(2 r_{1}+l\right) / 2$-thin, where

$$
r_{1}:=\max \{\operatorname{Arcsinh}(\operatorname{coth}(l / 4)), 4 \operatorname{Arcsinh}(1 / 2)\} .
$$

Remark. This is the best result that we can obtain about $Y$-pieces: If $L(\gamma) \leq l$ for one simple closed geodesic $\gamma \subseteq \partial Y_{0}, \delta$ can be arbitrarily long, as shows the example after the proof of Proposition 3.2.

Proof. Let us denote by $\gamma_{1}, \gamma_{2}, \gamma_{3}$, the simple closed geodesics in $\partial Y_{0}$ (as usual, we identify a puncture with a geodesic of zero length). Without loss of generality, we can assume that $L\left(\gamma_{3}\right)>l$, since if this was not so, we can apply Proposition 3.1 (we have $r_{1} \geq 2 r_{0}$ ).

If $\{i, j, k\}$ is any permutation of $\{1,2,3\}$, let us consider the geodesic $B_{i}$ in $Y_{0}$ which is orthogonal to $\gamma_{j}$ and $\gamma_{k}$. If we split $Y_{0}$ along the curves $B_{i}$, we obtain two isometric convex right-angle hexagons $H_{1}, H_{2}$.

For each point $p \in g_{3}:=\gamma_{3} \cap H_{1}$, we consider the geodesic $a_{p}$ in $H_{1}$ which starts in $p$ and finishes orthogonally to $B_{3}$. We want to obtain a bound for $L\left(a_{p}\right)$; in order to do this, let us consider first the simple case in which $\gamma_{1}$ and $\gamma_{2}$ are punctures. Then $H_{1}$ is a quadrilateral with two right angles and three sides of infinite length; if $p_{0}$ is the middle point of $g_{3}$, we can split $H_{1}$ by deleting $a_{p_{0}}$ in two isometric quadrilaterals $Q_{1}, Q_{2}$, with three right angles and two sides of infinite length. The other sides have length $L\left(\gamma_{3}\right) / 4$ and $L\left(a_{p_{0}}\right)$, with $\sinh \left(L\left(\gamma_{3}\right) / 4\right) \sinh L\left(a_{p_{0}}\right)=1$ (see e.g. [B, p. 157], [F, p. 89], [Ra, p. 96]). If $p_{0}, p_{1}$ are the end points of $g_{3}$ in $Q_{1}$, we can split $Q_{1}$ by deleting $a_{p_{1}}$ in a triangle and a quadrilateral $Q_{11}$ with three right angles and four finite sides. We have (see e.g. [F, p. 88]) $\sinh L\left(a_{p_{1}}\right)=\sinh L\left(a_{p_{0}}\right) \cosh \left(L\left(\gamma_{3}\right) / 4\right)=\operatorname{coth}\left(L\left(\gamma_{3}\right) / 4\right)$, and consequently $L\left(a_{p}\right) \leq L\left(a_{p_{1}}\right)<\operatorname{Arcsinh}(\operatorname{coth}(l / 4)) \leq r_{1}$, since $L\left(\gamma_{3}\right)>l$.

It is clear now that in the general case (with $0 \leq L\left(\gamma_{1}\right), L\left(\gamma_{2}\right) \leq l$ ) we have $L\left(a_{p}\right)<r_{1}+l / 2$.

Let us denote by $b_{p}$ the curve in $Y_{0}$ obtained by the union of $a_{p}$ and its symmetric curve $a_{p^{\prime}}$; each $b_{p}$ joins $\gamma_{3}$ with itself and have length less than $2 r_{1}+l$. It is clear that the set of the points of $Y_{0}$ which are not in the union of the $b_{p}$ 's has two connected components, which are tubular neighborhoods $N_{1}$ of $\gamma_{1}$ and $N_{2}$ of $\gamma_{2}$ in $Y_{0}$. It is plain that we can draw in $N_{i}$ curves freely homotopic to $\gamma_{i}$ with length less than $2 r_{1}+l$.

Then we have that $Y_{0}$ is $\left(2 r_{1}+l\right)$-decomposible and $3\left(2 r_{1}+l\right) / 2$-thin, by Theorem 2.1.

Example. The sharp hyperbolicity constant of the generalized $Y$-piece $Y_{t}$ with one puncture and two simple closed geodesics of length $2 t$ goes to infinity as $t \rightarrow \infty$. 
Let us denote by $\gamma_{1}, \gamma_{2}$, the simple closed geodesics of $Y_{t}$. The idea that lies behind the proof is that given two points in $\gamma_{i}$, the distance between them is approximately the length of a subcurve of $\gamma_{i}$ joining them. Let us denote by $p_{1} \in \gamma_{1}, p_{2} \in \gamma_{2}$, the points with $d\left(p_{1}, p_{2}\right)=d\left(\gamma_{1}, \gamma_{2}\right)=: s$. We choose the points $q_{1} \in \gamma_{1}, q_{2} \in \gamma_{2}$, with $d\left(p_{1}, q_{1}\right)=d\left(p_{2}, q_{2}\right)=t$. If we split $Y_{t}$ along the geodesics which start orthogonally to $\gamma_{1}$ in $p_{1}$ and $q_{1}$, and to $\gamma_{2}$ in $q_{2}$, we obtain two isometric right-angled hexagons $H_{1}, H_{2}$. Each $H_{i}$ has sides with length $t, s, t, \infty, 0, \infty$.

Standard hyperbolic trigonometry (see e.g. [B, p. 161]) gives

$$
\cosh s=1+\frac{2}{\sinh ^{2} t}, \quad \sinh s=\frac{2 \cosh t}{\sinh ^{2} t} .
$$

Let us consider the geodesic $\gamma_{0}$ in $H_{1}$ which gives the distance between $\left[p_{1}, q_{1}\right]$ and the side $A$ of infinite length which does not intersect with it; we define $x:=\gamma_{0} \cap\left[p_{1}, q_{1}\right]$ and $y:=\gamma_{0} \cap A$. The geodesic $\gamma_{0}$ splits $H_{1}$ into a right-angled pentagon and a quadrilateral with three right-angles and a degenerated angle. Hyperbolic trigonometry for pentagons (see e.g. [B, p. 159]) gives $\cosh L\left(\gamma_{0}\right)=\sinh s \sinh t=2 \operatorname{coth} t$; then $\lim _{t \rightarrow \infty} \cosh L\left(\gamma_{0}\right)=2$ and $\lim _{t \rightarrow \infty} \sinh L\left(\gamma_{0}\right)=\sqrt{3}$. We also have that $\sinh L\left(\gamma_{0}\right) \sinh d\left(x, q_{1}\right)=1$ and $\lim _{t \rightarrow \infty} d\left(x, q_{1}\right)=\operatorname{Arcsinh}(1 / \sqrt{3})$. Consequently, $d\left(x, q_{1}\right)$ is bounded when $t$ goes to $\infty$.

With this computations in mind, we consider the geodesic bigon $\gamma_{1}$ in $Y_{t}$ with vertices $\left\{p_{1}, q_{1}\right\}$ (it is geodesic by the symmetry of $\left.Y_{t}\right)$. Let us choose the point $z \in \gamma_{1} \cap H_{1}$ such that $d\left(z, p_{1}\right)=d\left(z, q_{1}\right)=t / 2$. Then there exists some constant $c$ with $d\left(z, \gamma_{1} \cap H_{2}\right) \geq t / 2-c$ if $t$ is large enough, as a consequence of the above computations; hence $\lim _{t \rightarrow \infty} \delta\left(Y_{t}\right)=\infty$.

In order to prove our next theorem, we need the following result appearing in [RT2]; in fact, Theorem 5.2 in [RT2] provides a better result than Theorem D, but this version is enough for our purpose.

Theorem D. ([RT2, Theorem 5.2]) Given an open non-exceptional Riemann surface S, let us denote by $F$ the union of some funnels of $S$. Let $S_{0}$ be the bordered non-exceptional Riemann surface obtained by deleting from $S$ the interior of $F$. Then $S$ is hyperbolic if and only if $S_{0}$ is hyperbolic. Furthermore, if $S_{0}$ is $\delta_{0}$-hyperbolic, then $S$ is $\delta$-hyperbolic, with $\delta$ a constant which only depends on $\delta_{0}$.

In order to state the next theorem we need a definition.

Definition 3.1. Let us consider a non-exceptional Riemann surface $S$ of finite type (with or without boundary); if $S$ is bordered, we also require that $\partial S$ is the union of local geodesics (closed or non-closed). An outer loop in $S$ is a simple closed geodesic which is the boundary curve of a funnel or is contained in $\partial S$. An inner loop in $S$ is a simple closed geodesic which is not an outer loop. The characteristic of $S$ is $a=2 g-2+n$, where $g$ is the genus of $S$ and $n$ is the sum of the number of punctures of $S$ and the number of outer loops of $S$.

We also need the following beautiful theorem of Bers.

Theorem E. ([Be, Theorem 1]) Let us consider a non-exceptional Riemann surface $S$ of finite type (with or without boundary); if $S$ is bordered, we also require that $\partial S$ is the union of local geodesics (closed or 
non-closed). If $S$ has characteristic a, the length of its shortest inner loop (if any) is bounded from above by a constant $J=J(a, L)$ depending only on $a$ and on the length $L$ of the longest outer loop (if any).

Remark. There exists such inner loop if $3 g-3+n>0$.

In fact, Theorem $\mathrm{E}$ is proved in [Be] only for surfaces without boundary, but the other case is direct from this one.

If we use Proposition 3.2 instead of Proposition 3.1 in the proof of Theorem 3.1, we obtain the following result.

Theorem 3.2. Let us consider a non-exceptional Riemann surface $S$ (with or without boundary) without genus. If there is a decomposition of $S$ into a union of funnels $\left\{F_{m}\right\}_{m \in M}$ and generalized $Y$-pieces $\left\{Y_{n}\right\}_{n \in N}$ $(N \neq \emptyset)$ with $L_{S}(\gamma) \leq l$ for at least two simple closed geodesics $\gamma \subseteq \partial Y_{n}$ for every $n \in N$, then $S$ is $\delta$-hyperbolic, where $\delta$ is a constant which only depends on $l$.

Proof. By Theorem D, we can assume that $M=\varnothing$. Let us consider $Y_{n}$ with $L_{S}(\gamma)>l$ for some simple closed geodesic $\gamma \subseteq \partial Y_{n}$. Let us assume that there exists some $n_{0} \neq n$ with $\gamma \subseteq \partial Y_{n_{0}}$; we can apply Theorem E to the bordered Riemann surface $Y_{n} \cup Y_{n_{0}}$ with characteristic $a=2$, and then we have an inner loop $\gamma^{\prime}$ in $Y_{n} \cup Y_{n_{0}}$ with $L_{S}\left(\gamma^{\prime}\right) \leq J(2, l)$. If we split $Y_{n} \cup Y_{n_{0}}$ by $\gamma^{\prime}$, we obtain two generalized $Y$-pieces $Y_{n}^{\prime}, Y_{n_{0}}^{\prime}$, such that $Y_{n} \cup Y_{n_{0}}=Y_{n}^{\prime} \cup Y_{n_{0}}^{\prime}$, and $L_{S}(\sigma) \leq l_{0}:=\max \{l, J(2, l)\}$ for every simple closed geodesic $\sigma \subseteq \partial Y_{n}^{\prime} \cup \partial Y_{n_{0}}^{\prime}$.

Consequently, without loss of generality, we can assume that the decomposition of $S$ in the union of generalized $Y$-pieces $\left\{Y_{n}\right\}_{n \in N}$ verifies the following property: if $L_{S}(\gamma)>l_{0}$ for some simple closed geodesic $\gamma \subseteq \cup_{n} \partial Y_{n}$, then $\gamma$ is in the boundary of just one generalized $Y$-piece.

By Proposition 3.2 we have that each $Y_{n}$ is $\left(2 r_{1}+l_{0}\right)$-decomposible, with

$$
r_{1}:=\max \left\{\operatorname{Arcsinh}\left(\operatorname{coth}\left(l_{0} / 4\right)\right), 4 \operatorname{Arcsinh}(1 / 2)\right\} .
$$

Since $S$ is a plane domain, the union in $n$ of the curves constructed in Proposition 3.2 in each $Y_{n}$ gives that $S$ is also $\left(2 r_{1}+l_{0}\right)$-decomposible, since any of such curves disconnects $S$. Consequently, Theorem 2.1 gives that $S$ is $3\left(2 r_{1}+l_{0}\right) / 2$-thin.

Since the funnel $F_{l}$ with $L\left(\partial F_{l}\right)=l$ has thin constant $\delta_{l} \geq l / 4$, one can think that a surface with funnels with arbitrarily long simple closed geodesics cannot be hyperbolic. However, Theorem 3.2 allows us to prove the following surprising result.

Corollary 3.1. There exist hyperbolic plane domains with funnels with arbitrarily long simple closed geodesics.

Proof. For each positive integer $n$ we consider a $Y$-piece $Y_{n}$ with two boundary geodesics of length 1 and a boundary geodesic of length $n$. We denote by $Z_{1}$ the union of $Y_{1}$ and two funnels with boundary geodesics of length 1 , and by $Z_{n}(n>1)$ the union of $Y_{n}$ and a funnel with boundary geodesic of length $n$. 
Let us denote by $\Omega$ the union of $\left\{Z_{n}\right\}_{n=1}^{\infty}$ identifying the boundary geodesics ( $Z_{1}$ is connected with $Z_{2}$, and $Z_{n}$ is connected with $Z_{n-1}$ and $Z_{n+1}$, if $n>1$ ). It is clear that $\Omega$ has funnels with arbitrarily long simple closed geodesics, and Theorem 3.2 gives that it is hyperbolic.

Definition 3.2. We say that a non-exceptional Riemann surface $S$ (with or without boundary) is of finite type if its fundamental group is finitely generated.

Definition 3.3. Let us consider a non-exceptional Riemann surface $S$ with boundary and $\left\{\eta_{n}^{1}, \eta_{n}^{2}\right\}_{n} \subseteq \partial S$ pairwise disjoint simple closed geodesics in $S$. If $c_{1}$ is a positive constant, we say that $\left\{\eta_{n}^{1}, \eta_{n}^{2}\right\}_{n}$ are $c_{1^{-}}$ identified if $L_{S}\left(\eta_{n}^{1}\right)=L_{S}\left(\eta_{n}^{2}\right) \leq c_{1}$ and $d_{S}\left(\eta_{n}^{1}, \eta_{n}^{2}\right) \leq c_{1}$ for every $n$.

If we apply Theorem 2.2 to the context of Riemann surfaces, we obtain the following result. It will be an important tool in the proof of Theorem 3.5.

Theorem 3.3. Let us consider a non-exceptional Riemann surface $S$ with boundary and $\left\{\eta_{n}^{1}, \eta_{n}^{2}\right\}_{n} c_{1^{-}}$ identified. Then $S$ is hyperbolic if and only if $S_{0}$ is hyperbolic. In particular, if $S$ (respectively $S_{0}$ ) is $\delta$-hyperbolic, then $S_{0}$ (respectively $S$ ) is $\delta^{\prime}$-hyperbolic, with $\delta^{\prime}$ a universal constant which only depends on $\delta$ and $c_{1}$.

Proof. This result is a direct consequence of Theorem 2.2 and the following facts:

Every non-exceptional Riemann surface (with or without boundary) is a geodesic metric space.

Since $\eta_{n}^{i}$ are simple closed curves, the condition that $\left(\eta_{n}^{1},\left.d_{S}\right|_{\eta_{n}^{1}}\right)$ and $\left(\eta_{n}^{2},\left.d_{S}\right|_{\eta_{n}^{2}}\right)$ are isometric is equivalent to $L_{S}\left(\eta_{n}^{1}\right)=L_{S}\left(\eta_{n}^{2}\right)$.

We have $\operatorname{diam}_{S}\left(\eta_{n}^{1} \cup \eta_{n}^{2}\right) \leq L_{S}\left(\eta_{n}^{1}\right) / 2+d_{S}\left(\eta_{n}^{1}, \eta_{n}^{2}\right)+L_{S}\left(\eta_{n}^{2}\right) / 2 \leq 2 c_{1}$.

If $\gamma_{1}, \gamma_{2}$, are disjoint simple closed geodesics contained in an open non-exceptional Riemann surface, with length less or equal than $a$, the Collar Lemma $[\mathrm{R}]$ says that there exist disjoint collars of $\gamma_{i}$ of width $d_{0}$, where $\cosh d_{0}=\operatorname{coth}(a / 2)$. Therefore, $d\left(\gamma_{1}, \gamma_{2}\right) \geq 2 \operatorname{Arccosh}(\operatorname{coth}(a / 2))$; it is clear that this inequality is also true if $S$ is bordered, since then $S$ is contained in an open non-exceptional Riemann surface.

In order to prove the next theorems we need some definitions.

Definition 3.4. Given a Riemann surface $S$ with finite genus $g$, we say that the simple closed geodesics $a_{1}, \ldots, a_{g}, b_{1}, \ldots, b_{g}$ are generators of the genus of $S$ if $S \backslash a_{j}$ and $S \backslash b_{j}$ are connected, $a_{j} \cap b_{j}$ is a single point, and $\left(a_{j} \cup b_{j}\right) \cap\left(\cup_{k \neq j}\left(a_{k} \cup b_{k}\right)\right)=\varnothing$.

Given $c>0$, we say that a Riemann surface $S$ with finite genus $g$ has c-small genus if there exist $a_{1}, \ldots, a_{g}, b_{1}, \ldots, b_{g}$ generators of the genus of $S$ such that $L_{S}\left(a_{j}\right) \leq c, L_{S}\left(b_{j}\right) \leq c$, for $j=1, \ldots, g$. We say that any plane domain (a surface without genus) has 0-small genus.

Definition 3.5. For each $l, c \geq 0$ and each non-negative integer $a$, we denote by $\mathcal{S}_{G}(a, l, c)$ the set of non-exceptional Riemann surfaces of finite type $S$ verifying the following properties: if $S$ is bordered, then $\partial S$ is the union of local geodesics (closed or non-closed), $S$ has characteristic less or equal than $a$ and $c$-small genus, and every outer loop has length less or equal than $l$.

We denote by $\mathcal{S}_{G}(a, l)$ the set of plane domains in $\mathcal{S}_{G}(a, l, c)$. 
and by $\mathcal{S}(a, l)$ the set of non-exceptional Riemann surfaces of finite type $S$ verifying the following properties: if $S$ is bordered, then the non-bounded components of $\partial S$ are local geodesics, $S$ has characteristic less or equal than $a$ and no genus, and every generalized outer loop has length less or equal than $l$.

The two following theorems guarantee the hyperbolicity of the surfaces of finite type, with hyperbolicity constants which only depend on just two or three topological and metric parameters.

Theorem 3.4. For each $l \geq 0$ and each non-negative integer $a$, there exists a constant $\delta=\delta(a, l)$, which only depends on a and $l$, such that every surface in $\mathcal{S}_{G}(a, l)$ is $\delta$-hyperbolic.

Proof. We prove the result by induction on $a$.

Let us consider first the case $a=0$. If $S \in \mathcal{S}_{G}(0, l)$, it is the punctured disk, an annulus or a funnel. Lemma 5.4 and Corollary 5.1 in [RT2] give the result for the punctured disk and the annuli; the case of the funnel is a consequence both of this fact and of the funnel being a geodesically convex subset of an annulus.

We consider now the case $a=1$. If $S \in \mathcal{S}_{G}(1, l)$, it is the union of a generalized $Y$-piece and at most three funnels. Since every simple closed geodesic of $S$ is an outer loop, Theorem 3.2 gives the result.

Let us assume now that the result is true for $a-1$, with $a \geq 2$, and let us prove it for $a$. Let us consider a surface $S \in \mathcal{S}_{G}(a, l)$. By Theorem E we can find an inner loop $\gamma$ with length less or equal than $J(a, l)$ (there exists such inner loop since $g=0$ and $a-1>0$ ). Then $S$ is the union of two surfaces $S_{1}, S_{2}$, with $S_{1} \cap S_{2}=\gamma$, since $S$ has genus 0 ; notice that $\gamma \subseteq \partial S_{1}, \partial S_{2}$. If we define $l_{a}:=J(a, l) / 2$ (which only depends on $a$ and $l), A_{1}:=A_{2}:=\varnothing$, we see that $\left\{S_{1}, S_{2}\right\}$ is a $\left(l_{a}, 0, l_{a}\right)$-tree-decomposition of $S$ (see Definition 2.8). It is clear that $S_{1}$ and $S_{2}$ have characteristic less than $a$, and every outer loop has length less or equal than $\max \{l, J(a, l)\}$; then we have that they are $\delta_{0}$-hyperbolic, with $\delta_{0}$ a constant which only depends on $a$ and $l$, by the induction hypothesis. Then Theorem $\mathrm{C}$ gives that there exists a constant $\delta=\delta(a, l)$, which only depends on $a$ and $l$, such that $S$ is $\delta$-hyperbolic.

We can improve this last result in the following theorem, in which we deal with the case of surfaces with genus.

Theorem 3.5. For each $l, c \geq 0$ and each non-negative integer $a$, there exists a constant $\delta=\delta(a, l, c)$, which only depends on $a, l$ and $c$, such that every surface in $\mathcal{S}_{G}(a, l, c)$ is $\delta$-hyperbolic.

Proof. Let us fix $a, l, c$, and let us consider $S \in \mathcal{S}_{G}(a, l, c)$. If $S \in \mathcal{S}_{G}(a, l)$, we only need to apply Theorem 3.4. If this was not so, we choose $a_{1}, \ldots, a_{g}, b_{1}, \ldots, b_{g}$ generators of the genus of $S$. Then we consider the bordered surface $S_{1}$ obtained by cutting $S$ along $a_{1}, \ldots, a_{g}$, and we define $t:=\max \{l, c\}$. To cut along $a_{j}$ decreases the genus by 1 and increases the number of outer loops by 2 ; therefore, the characteristic remains unchanged. It is clear that $S_{1} \in \mathcal{S}_{G}(a, t)$, and then we have by Theorem 3.4 that $S_{1}$ is $\delta_{0}$-hyperbolic, with $\delta_{0}$ a constant which only depends on $a$ and $t$. Notice that $L_{S}\left(b_{j}\right) \leq c$; hence the two copies of $a_{j}$ in $\partial S_{1}$ are $c$-identified; then Theorem 3.3 gives that there exists a constant $\delta=\delta\left(\delta_{0}, c\right)$, which only depends on $\delta_{0}$ and $c$, such that $S$ is $\delta$-hyperbolic. 
The conclusion of Theorem 3.5 is not true without the hypothesis of $c$-small genus, as shows by the following example:

Example. There exist open non-exceptional Riemann surfaces of finite type $S_{t}$ with genus 1 and characteristic 1, a puncture, and $\lim _{t \rightarrow 0^{+}} \delta\left(S_{t}\right)=\infty$ : For each $t>0$, let us consider the generalized $Y$-piece $Y_{t}$ with a puncture and two simple closed geodesics $\gamma_{1}, \gamma_{2}$, of length $2 t$. Splitting $Y_{t}$ into two isometric hexagons (with a side of zero length), standard hyperbolic trigonometry (see e.g. [B, p. 161]) gives

$$
d_{Y_{t}}\left(\gamma_{1}, \gamma_{2}\right)=\operatorname{Arccosh}\left(1+\frac{2}{\sinh ^{2} t}\right)=: g(t) .
$$

Let us denote by $S_{t}$ the Riemann surface of finite type with genus 1 and a puncture obtained from $Y_{t}$ by identifying $\gamma_{1}$ with $\gamma_{2}$. It is clear that there exists a simple closed geodesic with length $g(t)$ in $S_{t}$ "surrounding" the genus; then we have that if $S_{t}$ is $\delta$-thin, then $\delta \geq g(t) / 4$.

Theorems 3.5 and $\mathrm{C}$ give the following result.

Theorem 3.6. Let us consider a non-exceptional Riemann surface $S$ (with or without boundary). If there exists a tree-decomposition of $S$ into a union of bordered surfaces $\left\{S_{m}\right\}_{m \in M} \subset \mathcal{S}_{G}(a, l, c)$, then $S$ is $\delta$-hyperbolic, where $\delta$ is a constant which only depends on $a, l$ and $c$.

Remark. The condition " $\left\{S_{m}\right\}_{m \in M}$ is a tree-decomposition of $S$ " is verified if " $\left\{S_{m}\right\}_{m \in M}$ is a decomposition of $S$ such that for every $m, n \in M, \partial S_{m} \cap \partial S_{n}$ is the empty set or an outer loop $\gamma$ of $S_{m}$ and $S_{n}$, and $S \backslash \gamma$ is not connected if $\gamma=\partial S_{m} \cap \partial S_{n} "$; it is sufficient to take $k_{1}=l / 2, A_{n}=\varnothing, k_{2}=0$ and $k_{3}=(a+2) l / 2$, which are constants only depending on $a$ and $l$.

This remark and Theorem 3.6 give the following result.

Corollary 3.2. Let us consider a non-exceptional Riemann surface $S$ (with or without boundary) without genus. If there exists a decomposition of $S$ into a union of bordered surfaces $\left\{S_{m}\right\}_{m \in M} \subset \mathcal{S}_{G}(a, l)$, then $S$ is $\delta$-hyperbolic, where $\delta$ is a constant which only depends on a and $l$.

Now, we want to obtain the equivalence of the hyperbolicity of an extensive class of Riemann surfaces and some graphs. We start with a definition.

Definition 3.6. Let us consider a generalized $Y$-piece $Y_{0}$, with $L\left(\gamma_{i}\right)=l_{i} \leq l$, for every simple closed geodesic $\gamma_{i} \subseteq \partial Y_{0}$. We say that a tree $G:=(V, E)$ is the l-skeleton of $Y_{0}$ if $G$ has vertices $V=\left\{v, v_{1}, v_{2}, v_{3}\right\}$ and edges $E:=\cup_{i=1}^{3}\left[v, v_{i}\right]$, such that $L\left(\left[v, v_{i}\right]\right)=\operatorname{Arccosh}\left(\operatorname{coth}\left(l_{i} / 2\right)\right)$ for $i=1,2,3$.

Let us consider a generalized $Y$-piece $Y_{0}$, with $L\left(\gamma_{i}\right)=l_{i}$, for every simple closed geodesic $\gamma_{i} \subseteq \partial Y_{0}$, $l_{1}, l_{2} \leq l$ and $l_{3}>l$. We say that a tree $G$ is the $l$-skeleton of $Y_{0}$ if $G$ has just one edge $\left[v_{1}, v_{2}\right]$, such that

$$
L\left(\left[v_{1}, v_{2}\right]\right)=\operatorname{Arccosh}\left(\frac{\cosh \left(l_{3} / 2\right)+\cosh \left(l_{1} / 2\right) \cosh \left(l_{2} / 2\right)}{\sinh \left(l_{1} / 2\right) \sinh \left(l_{2} / 2\right)}\right) .
$$

Remark. If $L\left(\gamma_{i}\right)=l_{i}=0$ (i.e., if $\gamma_{i}$ is a puncture), we choose as $\left[v, v_{i}\right]$ a halfline starting in $v . L\left(\left[v_{1}, v_{2}\right]\right)$ is the distance between $\gamma_{1}$ and $\gamma_{2}$ (see e.g. [B, p. 161]). 
Proposition 3.3. Given any generalized $Y$-piece $Y_{0}$ with $L\left(\gamma_{i}\right)=l_{i} \leq l$, for at least two simple closed geodesic $\gamma_{i} \subseteq \partial Y_{0}$, there exists a $(1, M)$-quaiisometry of $Y_{0}$ onto its l-skeleton $G$, with

$$
M:=\max \left\{\frac{3}{2}\left(\log \left(2 \cosh \frac{l}{2}\left(1+\cosh \frac{l}{2}\right)\right)+\frac{l}{2} \operatorname{coth} \frac{l}{2}\right), 2 \operatorname{Arcsinh}\left(\operatorname{coth} \frac{l}{4}\right)+l\right\} .
$$

Proof. Let us denote by $\gamma_{1}, \gamma_{2}, \gamma_{3}$, the simple closed geodesics in $\partial Y_{0}$ (as usual, we identify a puncture with a geodesic of zero length).

We deal first with the case $L\left(\gamma_{i}\right)=l_{i} \leq l$, for $i=1,2,3$. The Collar Lemma gives that, for each geodesic $\gamma_{i}$, there exists a collar $C_{\gamma_{i}}$ of width $d_{i}=\operatorname{Arccosh}\left(\operatorname{coth}\left(l_{i} / 2\right)\right)$, with boundary curves $\gamma_{i}$ and $\eta_{i}$; the closed curve $\eta_{i}$ verifies $L\left(\eta_{i}\right)=L\left(\gamma_{i}\right) \cosh d_{i}=l_{i} \operatorname{coth}\left(l_{i} / 2\right)$ for $i=1,2,3$. When $\gamma_{i}$ is a puncture, we have $l_{i}=0$, $d_{i}=\infty$ and $L\left(\eta_{i}\right)=2$ (see [Bu, Chapter 4.4]).

If $\{i, j, k\}$ is any permutation of $\{1,2,3\}$, let us consider the geodesic segment $B_{i}$ in $Y_{0}$ which is orthogonal to $\gamma_{j}$ and $\gamma_{k}$. If we split $Y_{0}$ along the curves $B_{i}$, we obtain two isometric convex right-angle hexagons $H_{1}, H_{2}$, with consecutive sides of length $L\left(\gamma_{1}\right) / 2, L\left(B_{3}\right), L\left(\gamma_{2}\right) / 2, L\left(B_{1}\right), L\left(\gamma_{3}\right) / 2, L\left(B_{2}\right)$, such that

$$
L\left(B_{i}\right)=\operatorname{Arccosh}\left(\frac{\cosh \left(l_{i} / 2\right)+\cosh \left(l_{j} / 2\right) \cosh \left(l_{k} / 2\right)}{\sinh \left(l_{j} / 2\right) \sinh \left(l_{k} / 2\right)}\right)
$$

(see [B, p. 161]). Now, we define the hexagon $H_{1}^{*}:=H_{1} \backslash \cup_{i=1}^{3} C_{\gamma_{i}}$ in $H_{1}$ (similarly $H_{2}^{*}$ in $H_{2}$ ), with consecutive sides of length $L\left(\eta_{1}\right) / 2, \alpha_{3}, L\left(\eta_{2}\right) / 2, \alpha_{1}, L\left(\eta_{3}\right) / 2, \alpha_{2}$, verifying

$$
\begin{aligned}
\alpha_{i} & :=L\left(B_{i}\right)-\left(d_{j}+d_{k}\right) \\
& =\operatorname{Arccosh}\left(\frac{\cosh \left(l_{i} / 2\right)+\cosh \left(l_{j} / 2\right) \cosh \left(l_{k} / 2\right)}{\sinh \left(l_{j} / 2\right) \sinh \left(l_{k} / 2\right)}\right)-\left(\operatorname{Arccosh}\left(\operatorname{coth}\left(l_{j} / 2\right)\right)+\operatorname{Arccosh}\left(\operatorname{coth}\left(l_{k} / 2\right)\right)\right) \\
& \leq \log \left(2 \frac{\cosh \left(l_{i} / 2\right)+\cosh \left(l_{j} / 2\right) \cosh \left(l_{k} / 2\right)}{\sinh \left(l_{j} / 2\right) \sinh \left(l_{k} / 2\right)}\right)-\left(\log \left(\operatorname{coth}\left(l_{j} / 2\right)\right)+\log \left(\operatorname{coth}\left(l_{k} / 2\right)\right)\right) \\
& =\log \left(2 \frac{\cosh \left(l_{i} / 2\right)+\cosh \left(l_{j} / 2\right) \cosh \left(l_{k} / 2\right)}{\cosh \left(l_{j} / 2\right) \cosh \left(l_{k} / 2\right)}\right) \leq \log (2 \cosh (l / 2)(1+\cosh (l / 2))) .
\end{aligned}
$$

When $\gamma_{i}$ is a puncture, we obtain the same inequality by a limit process (see [Bu, Chapter 4.4]).

On the other hand, the function $g(t)=t \operatorname{coth}(t / 2)$ is increasing in $t \in(0, \infty)$; therefore $L\left(\eta_{i}\right) \leq l \operatorname{coth}(l / 2)$. Consequently, $L\left(\partial H_{i}^{*}\right) \leq 2 M$ and $\operatorname{diam}\left(H_{i}^{*}\right) \leq M$ for $i=1,2$.

Let us define $B_{2}^{\prime}:=B_{2} \cap C_{\gamma_{1}}, B_{3}^{\prime}:=B_{3} \cap C_{\gamma_{2}}$ and $B_{1}^{\prime}:=B_{1} \cap C_{\gamma_{3}}$.

We consider the continuous function $f: Y_{0} \longrightarrow G$, with $f\left(H_{1}^{*} \cup H_{2}^{*}\right)=v$, which is an isometry between $B_{2}^{\prime}$ and the edge $\left[v, v_{1}\right]$, between $B_{3}^{\prime}$ and $\left[v, v_{2}\right]$, and between $B_{1}^{\prime}$ and $\left[v, v_{3}\right]$. In the other points of $Y_{0}$, if $a \in C_{\gamma_{1}}$, we define $f(a)=f\left(a^{\prime}\right) \in\left[v, v_{1}\right]$, where $a^{\prime}$ is the point in $B_{2}^{\prime}$ such that $d\left(a, \gamma_{1}\right)=d\left(a^{\prime}, \gamma_{1}\right)$; we define $f$ in similar way in $C_{\gamma_{2}}$ and $C_{\gamma_{3}}$.

First of all, we have $d_{G}(f(x), f(y)) \leq d_{Y_{0}}(x, y)$ for every $x, y \in Y_{0}$.

We also have $d_{Y_{0}}(x, y) \leq d_{G}(f(x), f(y))+M$ for every $x, y \in Y_{0}$.

Therefore, we have that $f$ is a $(1, M)$-quasi-isometry of $Y_{0}$ onto $G$.

We deal now with the case $l_{1}, l_{2} \leq l$ and $l_{3}>l$. We have that $G=\left[v_{1}, v_{2}\right]$ and that $L\left(\left[v_{1}, v_{2}\right]\right)$ is equal to the length of the geodesic segment $B_{3}$ in $Y_{0}$ joining $\gamma_{1}$ and $\gamma_{2}$. We have seen in the proof of Proposition 3.2 that 
any point in $Y_{0}$ has a point of $B_{3}$ at distance less or equal than $\operatorname{Arcsinh}(\operatorname{coth}(l / 4))+l / 2 \leq M / 2$; consequently, the map $f_{1}: Y_{0} \longrightarrow B_{3}$ such that $f_{1}(x)$ is the nearest point to $x$ in $B_{3}$ verifies $d_{B_{3}}\left(f_{1}(x), f_{1}(y)\right) \leq d_{Y_{0}}(x, y) \leq$ $d_{B_{3}}\left(f_{1}(x), f_{1}(y)\right)+M$ for every $x, y \in Y_{0}$. Therefore, we have that $f:=f_{2} \circ f_{1}$ is a $(1, M)$-quasi-isometry of $Y_{0}$ onto $G$, if $f_{2}$ is an isometry between $B_{3}$ and $G$.

Definition 3.7. Let us consider $l>0$ and a Riemann surface $S$ (with or without boundary), such that there is a decomposition of $S$ into a union of generalized $Y$-pieces $\left\{Y_{n}\right\}_{n \in N}$ and funnels $\left\{F_{m}\right\}_{m \in M}$, with $L_{S}(\gamma) \leq l$ for at least two simple closed geodesics $\gamma \subseteq \partial Y_{n}$ in each $n$. We say that a graph $G$ is a $l$-skeleton of $S$ if it is the union of $\left\{G_{n}\right\}_{n \in N}$ with the following properties:

(a) $G_{n}$ is the $l$-skeleton of $Y_{n}$ for $n \in N$.

(b) If $Y_{n} \cap Y_{m}=\cup_{i \in I_{n m}} \gamma_{n m}^{i}$ (with $\gamma_{n m}^{i}=\gamma_{m n}^{i}$ ), then $G_{n} \cap G_{m}=\cup_{i \in I_{n m}} v_{n m}^{i}$, where $v_{n m}^{i}$ is the vertex associated to $\gamma_{n m}^{i}$, and we identify $v_{n m}^{i}$ with $v_{m n}^{i}$ in order to obtain $G$.

Remarks. 1. As a consequence of (b), we have:

(P) if $L_{S}(\gamma)>l$ for some simple closed geodesic $\gamma \subseteq \cup_{n} \partial Y_{n}$, then $\gamma$ is in the boundary of just one generalized $Y$-piece.

We want to remark that $(\mathrm{P})$ is not a restriction at all, since if $\left\{Y_{n}\right\}_{n}$ does not satisfy this property, we can change $\left\{Y_{n}\right\}_{n}$ by $\left\{Y_{n}^{\prime}\right\}_{n}$, as in the proof of Theorem 3.2, such that $\left\{Y_{n}^{\prime}\right\}_{n}$ verifies (P) with $\max \{l, J(2, l)\}$ instead of $l$. Consequently, if $S$ has a decomposition into a union of generalized $Y$-pieces $\left\{Y_{n}\right\}_{n \in N}$ and funnels $\left\{F_{m}\right\}_{m \in M}$, with $L_{S}(\gamma) \leq l$ for at least two simple closed geodesics $\gamma \subseteq \partial Y_{n}$ in each $n$, then it has a $\max \{l, J(2, l)\}$-skeleton.

2. Notice that card $I_{n m} \leq 3$, and $G_{n} \cap G_{m}=\varnothing$ if and only if $Y_{n} \cap Y_{m}=\varnothing$.

Theorem 3.7 below let us move the study of the hyperbolicity of a Riemann surface $S$ to a graph $G$ with much simpler structure, so long as between them there exists the precise relationship described in Definition 3.7 .

Theorem 3.7. Let us consider a non-exceptional Riemann surface $S$ (with or without boundary), with a l-skeleton $G$. If $S$ (respectively $G$ ) is $\delta$-hyperbolic, then $G$ (respectively $S$ ) is $\delta^{\prime}$-hyperbolic, with $\delta^{\prime}$ a constant which only depends on $\delta$ and $l$.

Proof. Without loss of generality, we can assume that $S$ does not have funnels by Theorem D. We see now that there exists a $(1+c, 2 M)$-quaiisometry of $S$ onto $G$, with $M$ the constant in Proposition 3.3 and $c:=M /(2 \operatorname{Arccosh}(\operatorname{coth}(l / 2)))$.

For each $n \in N$, we have $\partial Y_{n}=\cup_{m i} \gamma_{n m}^{i}$ (as usual, we identify a puncture with a geodesic of zero length). Let us consider the $(1, M)$-quasi-isometry $f_{n}: Y_{n} \longrightarrow G_{n}$ defined in the proof of Proposition 3.3. Let us define $f: S \longrightarrow G$ such that $\left.f\right|_{Y_{n}}:=f_{n}$; we will show now that $f$ is a $(1+c, 2 M)$-quasi-isometry.

First of all, we have that $d_{G}(f(x), f(y)) \leq d_{S}(x, y)$ for every $x, y \in S$.

We prove now the reverse inequality. If $x$ and $y$ are in the same $Y_{n}$, we apply Proposition 3.3. If $x$ and $y$ are not in the same $Y_{n}$, let us consider a geodesic $g$ joining $f(x)$ and $f(y)$ in $G$. Since $g$ meets at most a finite number of $G_{n}$ 's, we denote them by $G_{n_{1}}, G_{n_{2}}, \ldots, G_{n_{r}}$, where $f(x) \in G_{n_{1}}, f(y) \in G_{n_{r}}$, and $g$ meets 
$G_{n_{k+1}}$ after $G_{n_{k}}$. Now, we take a continuous curve $\gamma$ in $S$ joining $x$ and $y$, such that $f(\gamma)=g$ and $\gamma \cap Y_{n}$ is a geodesic in $Y_{n}$; then $\gamma$ meets each simple closed curve $\sigma \subseteq \cup_{n} \partial Y_{n}$ at most in a point, $\gamma$ only meets the pieces $Y_{n_{1}}, Y_{n_{2}}, \ldots, Y_{n_{r}}$, and $\gamma$ meets $Y_{n_{k+1}}$ after $Y_{n_{k}}$.

First of all, recall that $d_{G_{n}}\left(v_{n m_{1}}^{i_{1}}, v_{n m_{2}}^{i_{2}}\right) \geq 2 \operatorname{Arccosh}(\operatorname{coth}(l / 2))$, by the Collar Lemma. Consequently, if $a \in \gamma_{n m_{1}}^{i_{1}}, b \in \gamma_{n m_{2}}^{i_{2}}$, we obtain (using Proposition 3.3)

$$
d_{Y_{n}}(a, b) \leq d_{G_{n}}\left(v_{n m_{1}}^{i_{1}}, v_{n m_{2}}^{i_{2}}\right)+M=d_{G_{n}}\left(v_{n m_{1}}^{i_{1}}, v_{n m_{2}}^{i_{2}}\right)+2 c \operatorname{Arccosh}(\operatorname{coth}(l / 2)) \leq(1+c) d_{G_{n}}\left(v_{n m_{1}}^{i_{1}}, v_{n m_{2}}^{i_{2}}\right) .
$$

If we define $x_{k}:=\gamma \cap \partial Y_{n_{k}} \cap \partial Y_{n_{k+1}}$, for $k=1, \ldots, r-1$, we have

$$
\begin{aligned}
d_{S}(x, y) & \leq L_{S}(\gamma)=d_{Y_{n_{1}}}\left(x, x_{1}\right)+\sum_{k=2}^{r-1} d_{Y_{n_{k}}}\left(x_{k-1}, x_{k}\right)+d_{Y_{n_{r}}}\left(x_{r-1}, y\right) \\
& \leq d_{G_{n_{1}}}\left(f(x), f\left(x_{1}\right)\right)+M+(1+c) \sum_{k=2}^{r-1} d_{G_{n_{k}}}\left(f\left(x_{k-1}\right), f\left(x_{k}\right)\right)+d_{G_{n_{r}}}\left(f\left(x_{r-1}\right), f(y)\right)+M \\
& \leq(1+c) d_{G}(f(x), f(y))+2 M .
\end{aligned}
$$

Therefore, $f: S \longrightarrow G$ is a $(1+c, 2 M)$-quasi-isometry, and Theorem B finishes the proof.

Next we prove that the hyperbolicity is stable under significant metric changes (even with non-quasiisometric deformations), as long as the topology is preserved. The following definition describes the outstanding parameters involved in the kind of deformations studied in Theorem 3.8.

Definition 3.8. Given a positive constant $l$, we say that two Riemann surfaces $S$ and $S^{\prime}$ (with or without boundary) have similar l-skeletons if there are decompositions $\left\{Y_{n}\right\}_{n \in N} \cup\left\{F_{m}\right\}_{m \in M}$ of $S$ and $\left\{Y_{n}^{\prime}\right\}_{n \in N} \cup\left\{F_{m}^{\prime}\right\}_{m \in M^{\prime}}$ of $S^{\prime}$, with associated $l$-skeletons $G$ and $G^{\prime}$ respectively, such that:

(a) $Y_{n} \cap Y_{m}=\cup_{i \in I_{n m}} \gamma_{n m}^{i}$ (with $\gamma_{n m}^{i}=\gamma_{m n}^{i}$ ) and $Y_{n}^{\prime} \cap Y_{m}^{\prime}=\cup_{i \in I_{n m}} \eta_{n m}^{i}$ (with $\eta_{n m}^{i}=\eta_{m n}^{i}$ ).

(b) If we define $c_{1}:=\inf \left\{L_{S}(\gamma): \gamma \subseteq\left(\cup_{n} \partial Y_{n}\right) \backslash \partial S\right.$ and $S \backslash \gamma$ is connected $\}$ and $c_{1}^{\prime}:=\inf \left\{L_{S^{\prime}}(\eta): \eta \subseteq\right.$ $\left(\cup_{n} \partial Y_{n}^{\prime}\right) \backslash \partial S^{\prime}$ and $S^{\prime} \backslash \eta$ is connected $\}$, then $c_{1}=0$ if and only if $c_{1}^{\prime}=0$.

(c) If we define $c_{2}:=\sup \left\{L_{S}(\gamma): \gamma \subseteq\left(\cup_{m} \partial F_{m}\right) \cup \partial S, \gamma \subseteq \partial Y_{n}\right.$ and $S \backslash Y_{n}$ is connected $\}$ and $c_{2}^{\prime}:=$ $\sup \left\{L_{S^{\prime}}(\eta): \eta \subseteq\left(\cup_{m} \partial F_{m}^{\prime}\right) \cup \partial S^{\prime}, \eta \subseteq \partial Y_{n}^{\prime}\right.$ and $S^{\prime} \backslash Y_{n}^{\prime}$ is connected $\}$, then $c_{2}=\infty$ if and only if $c_{2}^{\prime}=\infty$.

Theorem 3.8. Let us consider two non-exceptional Riemann surfaces $S$ and $S^{\prime}$ (with or without boundary) with similar l-skeletons. Then $S$ is hyperbolic if and only if $S^{\prime}$ is hyperbolic. Furthermore, if $S$ is $\delta$-hyperbolic, then $S^{\prime}$ is $\delta^{\prime}$-hyperbolic, with $\delta^{\prime}$ a constant which only depends on $\delta, l, c_{j}$ and $c_{j}^{\prime}(j=1,2)$.

Proof. Without loss of generality, we can assume that $S$ and $S^{\prime}$ do not have funnels, by Theorem D. If $c_{1}=0$, then there exist geodesics $\gamma_{n m}^{i}$ (which do not disconnect $S$ ), with lengths $l_{n m}^{i} \rightarrow 0$; then Theorem 3.3 in [PRT2] gives that $S$ is not hyperbolic (since $c_{1}^{\prime}=0$, we also have that $S^{\prime}$ is not hyperbolic).

If $c_{2}=\infty$, then there exist generalized $Y$-pieces $Y_{n}$ (which do not disconnect $S$ ), with $l_{n m_{1}}^{i_{1}}, l_{n m_{2}}^{i_{2}} \leq l$ and $d_{Y_{n}}\left(\gamma_{n m_{1}}^{i_{1}}, \gamma_{n m_{2}}^{i_{2}}\right) \rightarrow \infty$; then Theorem 2.2 in [PRT2] gives that $S$ is not hyperbolic (since $c_{2}^{\prime}=\infty$, we also have that $S^{\prime}$ is not hyperbolic).

Let us assume now that $c_{1}, c_{1}^{\prime}>0$ and $c_{2}, c_{2}^{\prime}<\infty$. First, we prove the result if $S \backslash \gamma$ and $S^{\prime} \backslash \eta$ are connected for every $\gamma \subseteq \cup_{n} \partial Y_{n}$ and $\eta \subseteq \cup_{n} \partial Y_{n}^{\prime}$. If $G$ and $G^{\prime}$ are the $l$-skeletons of $S$ and $S^{\prime}$ respectively, 
Theorem 3.7 gives that there exist two surjective $(1+c, 2 M)$-quasi-isometries $f: S \longrightarrow G$ and $f^{\prime}: S^{\prime} \longrightarrow G^{\prime}$, where $M$ and $c$ only depend on $l$. By Theorem B, we only need to prove that if $G$ is $\delta_{0}$-hyperbolic, then $G^{\prime}$ is $\delta_{0}^{\prime}$-hyperbolic, with $\delta_{0}^{\prime}$ a constant which only depends on $\delta_{0}, l, c_{j}$ and $c_{j}^{\prime}$ (since $S$ and $S^{\prime}$ play symmetric roles).

We say that an edge $e$ in a graph is a leaf if a vertex of $e$ has degree one. Now, let us consider the graph $G_{0}$ (respectively $G_{0}^{\prime}$ ) obtained by removing from $G$ (respectively $G^{\prime}$ ) its set of leaves. Let us remark that $\delta\left(G_{0}\right)=\delta(G)$ and $\delta\left(G_{0}^{\prime}\right)=\delta\left(G^{\prime}\right)$.

We define a function $F: G_{0} \longrightarrow G_{0}^{\prime}$, in the following way:

Let us consider a generalized $Y$-piece $Y_{n}$ such that its three curves in $\partial Y_{n}$ have length less or equal than l. If $f\left(\gamma_{n m}^{i}\right)=v_{n m}^{i}$ and $f^{\prime}\left(\eta_{n m}^{i}\right)=w_{n m}^{i}, F$ is a dilatation of $\left[v_{n}, v_{n m}^{i}\right] \in G_{0}$ onto $\left[w_{n}, w_{n m}^{i}\right] \in G_{0}^{\prime}$.

If $Y_{n}$ has a curve in $\partial Y_{n}$ with length greater than $l$, and the other boundary curves are $\gamma_{n m_{1}}^{i_{1}}, \gamma_{n m_{2}}^{i_{2}}, F$ is a dilatation of $\left[v_{n m_{1}}^{i_{1}}, v_{n m_{2}}^{i_{2}}\right] \in G_{0}$ onto $\left[w_{n m_{1}}^{i_{1}}, w_{n m_{2}}^{i_{2}}\right] \in G_{0}^{\prime}$.

Let us prove now that $F$ is a bijective $(\alpha, 0)$-quasi-isometry, beeing $\alpha$ a constant which only depends on $l, c_{j}$ and $c_{j}^{\prime}$ : Since $c_{1}, c_{1}^{\prime}>0$ and $c_{2}, c_{2}^{\prime}<\infty$, and there are no leaves either in $G_{0}$ or in $G_{0}^{\prime}$, then $c_{1} \leq l_{n m}^{i}:=L_{S}\left(\gamma_{n m}^{i}\right) \leq \max \left\{l, c_{2}\right\}$ and $c_{1}^{\prime} \leq L_{n m}^{i}:=L_{S^{\prime}}\left(\eta_{n m}^{i}\right) \leq \max \left\{l, c_{2}^{\prime}\right\}$, if $v_{n m}^{i} \in G_{0}$ (recall that $v_{n m}^{i} \in G_{0}$ if and only if $\left.w_{n m}^{i} \in G_{0}^{\prime}\right)$. Hence $L_{G}\left(\left[v_{n}, v_{n m}^{i}\right]\right)=\operatorname{Arccosh}\left(\operatorname{coth}\left(l_{n m}^{i} / 2\right)\right)$ and $L_{G^{\prime}}\left(\left[w_{n}, w_{n m}^{i}\right]\right)=$ $\operatorname{Arccosh}\left(\operatorname{coth}\left(L_{n m}^{i} / 2\right)\right)$ are comparable with constants which only depend on $l, c_{j}$ and $c_{j}^{\prime}$ (if every curve in $\partial Y_{n}$ has length less or equal than $l$ ). The same is true for $L_{G}\left(\left[v_{n m_{1}}^{i_{1}}, v_{n m_{2}}^{i_{2}}\right]\right)$ and $L_{G^{\prime}}\left(\left[w_{n m_{1}}^{i_{1}}, w_{n m_{2}}^{i_{2}}\right]\right.$ ) (if $Y_{n}$ has a curve in $\partial Y_{n}$ with length greater than $l$ ).

Let us assume now that there are geodesics $\gamma_{n m}^{i}$ such that $S \backslash \gamma_{n m}^{i}$ is not connected (then we also have $S^{\prime} \backslash \eta_{n m}^{i}$ is not connected). In this case, we can decompose $S=\cup_{r} S_{r}$ (respectively $S^{\prime}=\cup_{r} S_{r}^{\prime}$ ), where $\left\{S_{r}\right\}_{r}$ are the connected components which we obtain by splitting $S$ for every simple closed geodesic $\gamma \subseteq \cup_{n} \partial Y_{n}$ with $S \backslash \gamma$ not connected; then any simple closed geodesic $\gamma \subseteq\left(\cup_{n} \partial Y_{n}\right) \cap S_{r}$ (respectively $\left.\eta \subseteq\left(\cup_{n} \partial Y_{n}^{\prime}\right) \cap S_{r}^{\prime}\right)$ does not disconnect $S_{r}$ (respectively $S_{r}^{\prime}$ ). Consequently $\left\{S_{r}\right\}_{r}$ is a $\left(k_{1}, k_{2}, 0\right)$-tree-decomposition of $S$ with $A_{n}=\Lambda, k_{1}=\frac{l}{2}$ and $k_{2}=\frac{l}{4 \operatorname{Arccosh}(\operatorname{coth}(l / 2))}$ (see Definition 2.8; in order to estimate $d_{S_{r}}\left(\gamma_{n m}^{i}, \gamma_{s t}^{u}\right)$ we can use the Collar Lemma, since $\gamma_{n m}^{i}, \gamma_{s t}^{u}$ are disjoint simple closed geodesics). Similarly $\left\{S_{r}^{\prime}\right\}_{r}$ is also a $\left(k_{1}, k_{2}, 0\right)$-tree-decomposition of $S^{\prime}$.

Then Theorem C gives that if $S$ is $\delta$-hyperbolic then $S_{r}$ is $\delta_{1}$-hyperbolic for every $r$, with $\delta_{1}$ a constant which only depends on $\delta$ and $l$. Now, we can apply the last argument to $S_{r}$ and $S_{r}^{\prime}$, and therefore $S_{r}^{\prime}$ is $\delta_{2}$-hyperbolic with $\delta_{2}$ a constant which only depends on $\delta, l, c_{j}$ and $c_{j}^{\prime}$. Finally, we use again Theorem $\mathrm{C}$ to assure that $S^{\prime}$ is $\delta^{\prime}$-hyperbolic, with $\delta^{\prime}$ only depending on $\delta, l, c_{j}$ and $c_{j}^{\prime}$.

This finishes the proof because of the symmetry between $S$ and $S^{\prime}$.

Remark. After the proofs of theorems 3.7 and 3.8, it is clear that the conclusions of these theorems also hold if we define the $l$-skeleton of a $Y$-piece in the following similar way:

If $L\left(\gamma_{i}\right)=l_{i} \leq l$, for $i=1,2,3$, we define $L\left(\left[v, v_{i}\right]\right):=\log \left(1+l_{i}^{-1}\right)$ for $i=1,2,3$. If $l_{1}, l_{2} \leq l$ and $l_{3}>l$, we take $L\left(\left[v_{1}, v_{2}\right]\right):=\log \left(1+l_{1}^{-1}\right)+\log \left(1+l_{2}^{-1}\right)+l_{3}$. 
As a consequence of Theorem 3.8, we obtain that hyperbolicity is a property stable under "twisting", for Riemann surfaces with $l$-skeletons (the result is not true without this hypothesis).

Notice that if two non-exceptional Riemann surfaces have the same $l$-skeleton $G$, they have the same decomposition $\left\{Y_{n}\right\}_{n \in N} \cup\left\{F_{m}\right\}_{m \in M}$, and they are obtained by gluing the pieces following the same design of $G$, after applying a twist to the curves in $\cup_{n} \partial Y_{n}$.

Corollary 3.3. Let us consider two non-exceptional Riemann surfaces $S, S^{\prime}$ (with or without boundary), with the same l-skeleton. If $S$ is $\delta$-hyperbolic, then $S^{\prime}$ is $\delta^{\prime}$-hyperbolic, with $\delta^{\prime}$ a constant which only depends on $\delta$ and $l$.

\section{The hyperbolicity in the Classification Theory of Riemann surfaces}

We prove in this section that there is no inclusion relationship between hyperbolic Riemann surfaces and the usual classes of Riemann surfaces, such as $O_{G}$ (surfaces without Green's function), surfaces with hyperbolic isoperimetric inequality, or the complements of these classes (even in the case of plane domains). This fact is important since it points out that hyperbolic Riemann surfaces are a class completely different from the more usual classes of Riemann surfaces. This makes the study of hyperbolic Riemann surfaces more complicated and interesting.

We have the same result for the classes $O_{H P}$ (surfaces without non-constant harmonic positive functions), $O_{H B}$ (surfaces without non-constant harmonic bounded functions), and $O_{H D}$ (surfaces without non-constant harmonic functions with finite Dirichlet integral), since in the case of plane domains (and even for surfaces with finite genus) we have $O_{G}=O_{H P}=O_{H B}=O_{H D}$ (see e.g. [AS, p. 208]).

Let us denote by $H$ the class of hyperbolic Riemann surfaces and by $H I I$ the class of Riemann surfaces with hyperbolic isoperimetric inequality. We are going to provide some plane domains which can be used so as to prove the following facts:

(a) $H I I$ is not contained in $H$.

(b) $(H I I)^{c}$ is not contained in $H$.

(c) $H$ is not contained in $H I I$.

(d) $H$ is not contained in $(H I I)^{c}$.

(e) $O_{G}$ is not contained in $H$.

(f) $\left(O_{G}\right)^{c}$ is not contained in $H$.

(g) $H$ is not contained in $O_{G}$.

(h) $H$ is not contained in $\left(O_{G}\right)^{c}$.

Notice that we only need to prove (a), (d), (e) and (h), since $H I I \subset\left(O_{G}\right)^{c}$.

Definition 4.1. We can define the modulus of an annulus $\left\{r<\left|z-z_{0}\right|<R\right\}$ as $R / r$. We say that the annulus $A=\left\{r<\left|z-z_{0}\right|<R\right\}$ separates the boundary of the plane domain $\Omega$ if $A \subseteq \Omega$ and each connected component of the complement of $A$ meets $\partial \Omega$. We say that a plane domain $\Omega$ is modulated if there is an upper bound for the modulus of every annulus which separates the boundary of $\Omega$. 
Any modulated plane domain belongs to $H I I$ (see e.g. [FR1, Theorem 3]). Let us recall that a plane domain $\Omega$ belongs to $O_{G}$ if and only if $\partial \Omega$ has zero logarithmic capacity (see e.g. [AS, p. 249]). Hence, the plane domains in $O_{G}$ (and in $O_{H P}, O_{H B}, O_{H D}$ ) can be characterized by the size of their boundaries. However, when we deal with hyperbolic plane domains the situation is much more sophisticated.

(a) There exists a plane domain $\Omega_{1} \in H I I \cap H^{c}$.

For each natural number $n$ we consider a real number $a_{0}:=0$ (if $n=0$ ) and $a_{n} \in(0,1 / 2)$ (if $n>0$ ), the sets $E_{n}:=\{|z-2 n|=1 / 2\}, F_{n}:=\left\{|z-2 n|=1 / 2+1 /(n+2),|\operatorname{Im} z| \geq a_{n}, \operatorname{Re} z \leq 2 n\right\}$ and $G_{n}:=\left\{|z-2 n|=1 / 2+1 /(n+2),|\operatorname{Im} z| \geq a_{n+1}, \operatorname{Re} z \geq 2 n\right\}$. Let us denote by $H_{n}^{+}$the segment contained in $\left\{\operatorname{Im} z=a_{n+1}\right\}$ joining the point $G_{n} \cap\left\{\operatorname{Im} z=a_{n+1}\right\}$ with $F_{n+1} \cap\left\{\operatorname{Im} z=a_{n+1}\right\}$; we denote by $H_{n}^{-}$the conjugated of $H_{n}^{+}$.

We define $\Omega_{1}$ as the unique plane domain whose boundary is equal to $\cup_{n}\left(E_{n} \cup F_{n} \cup G_{n} \cup H_{n}^{+} \cup H_{n}^{-}\right)$.

We have that $\Omega_{1} \in H I I$ since it is a modulated domain.

If we denote by $\gamma_{n}$ the simple closed geodesic in $\Omega_{1}$ freely homotopic to the ideal boundary $E_{n}$, we choose the sequence $\left\{a_{n}\right\}_{n}$ small enough to guarantee that $\gamma_{n} \cap\{\operatorname{Im} z \geq 0\}$ is a geodesic in $\Omega_{1}$. Then, for each $n$, we can choose a geodesic triangle contained in $\gamma_{n}$ with thin constant greater or equal than $L\left(\gamma_{n}\right) / 4$. Since $L\left(\gamma_{n}\right) \longrightarrow \infty$, we deduce that $\Omega_{1}$ is not hyperbolic.

(d) The unit disk is a plane domain contained in $H \cap H I I$.

(e) There exists a plane domain $\Omega_{2} \in O_{G} \cap H^{c}$.

For each positive integer $n$ we consider isometric generalized $Y$-pieces $\left\{Y_{n}^{r}\right\}_{r=1}^{2 N_{n}}$ with two boundary geodesics of length $n$ and a puncture. Let us denote by $Z_{n}$ the union of $\left\{Y_{n}^{r}\right\}_{r=1}^{2 N_{n}}$ identifying the boundary geodesics (we join $Y_{n}^{r}$ with $Y_{n}^{r-1}$ and $Y_{n}^{r+1}$, if $1<r<2 N_{n}$ ); we paste these pieces without "twist", i.e. if $\alpha_{n}^{r}$ is the geodesic joining the two boundary geodesics of $Y_{n}^{r}$, we identify a point of $\alpha_{n}^{r}$ with a point of $\alpha_{n}^{r+1}$.

We denote by $Y_{1}$ the generalized $Y$-piece with two punctures and a boundary geodesic of length 1; for each positive integer $n>1$ we consider a generalized $Y$-piece $Y_{n}$ with a puncture and two boundary geodesics of length $n$ and $n-1$.

Let us define $\Omega_{2}$ as the union of $\left\{Z_{n}\right\}_{n=1}^{\infty}$ and $\left\{Y_{n}\right\}_{n=1}^{\infty}$ identifying boundary geodesics of equal length (we join $Z_{n}$ with $Y_{n}$ and $Y_{n+1}$ without "twist").

We denote by $\beta_{n}$ the geodesic $\beta_{n}:=\cup_{n=1}^{2 N_{n}} \alpha_{n}$ joining the two boundary geodesics of $Z_{n}$.

Let us consider the "central" geodesic of $Z_{n}, \gamma_{n}:=Y_{n}^{N_{n}} \cap Y_{n}^{N_{n}+1}$. The symmetry of $Z_{n}$ guarantee that there is a geodesic bigon (see the remark after Definition 2.3) in $\Omega_{2}$ contained in $\gamma_{n}$ : we choose as vertices $u_{n}:=\beta_{n} \cap \gamma_{n}=\alpha_{n}^{N_{n}} \cap \alpha_{n}^{N_{n}+1}$ and $v_{n} \in \gamma_{n}$ with $d_{\gamma_{n}}\left(u_{n}, v_{n}\right)=n / 2$. Let us choose $N_{n}$ large enough in order to have $d_{Z_{n}}\left(\gamma_{n}, \partial Z_{n}\right) \geq n$; this inequality gives that this bigon has sharp thin-constant equal to $n / 4$. Hence, $\Omega_{2}$ is not hyperbolic.

It is not very difficult to see that $\Omega_{2}$ is in $O_{G}$ : Let us consider the simple closed geodesic $\sigma_{n}$ that joins $Y_{n}$ with $Z_{n}, \Phi_{n}^{r}$ the family of curves joining the two simple closed geodesics in $Y_{n}^{r}$, and $\Gamma_{n}$ the family of curves joining $\sigma_{1}$ with $\sigma_{n+1}$ in $\cup_{j=1}^{n-1}\left(Z_{j} \cup Y_{j+1}\right)$. In order to see that $\Omega_{2} \in O_{G}$, it is sufficient to see that 
$\lim _{n \rightarrow \infty} \Lambda\left(\Gamma_{n}\right)=\infty$, by [AS, p. 229], where $\Lambda\left(\Gamma_{n}\right)$ denotes the extremal length of $\Gamma_{n}$ (see [AS, pp. 220$223]$ for the definition and properties of extremal length). Since $\Lambda\left(\Phi_{j}^{r}\right)$ does not depend on $r$, the second theorem in [AS, p. 222] gives that $\Lambda\left(\Gamma_{n}\right) \geq \sum_{j=1}^{n-1} 2 N_{j} \Lambda\left(\Phi_{j}^{r}\right)$. If we choose $N_{j} \geq 1 / \Lambda\left(\Phi_{j}^{r}\right)$, we obtain $\lim _{n \rightarrow \infty} \Lambda\left(\Gamma_{n}\right) \geq \lim _{n \rightarrow \infty}(2 n-2)=\infty$, and consequently $\Omega_{2} \in O_{G}$.

(h) The twice puncture plane $\mathbf{C} \backslash\{0,1\}$ (the generalized $Y$-piece with three punctures) is a plane domain contained in $H \cap O_{G}$. It is hyperbolic by Proposition 3.1, since it is a generalized $Y$-piece, and it is in $O_{G}$ since a finite number of points has zero logarithmic capacity.

\section{REFERENCES}

[1] Alvarez, V., Rodríguez, J. M., Structure theorems for Riemann and topological surfaces, J. London Math. Soc. 69 (2004), 153-168.

[2] Alvarez, V., Rodríguez, J.M. and Yakubovich, V.A., Subadditivity of p-harmonic "measure" on graphs, Michigan Mathematical Journal 49 (2001), 47-64.

[3] Anderson, J. W., Hyperbolic Geometry. Springer, London, 1999.

[4] Balogh, Z. M., Buckley, S. M., Geometric characterizations of Gromov hyperbolicity, Invent. Math. 153 (2003), 261-301.

[5] Beardon, A. F., The geometry of discrete groups. Springer-Verlag, New York, 1983.

[6] Bonk, M., Quasi-geodesics segments and Gromov hyperbolic spaces, Geometriae Dedicata 62 (1996), 281-298.

[7] Bonk, M., Heinonen, J., Koskela, P., Uniformizing Gromov hyperbolic spaces. Astérisque No. 270 (2001).

[8] Buser, P., Geometry and Spectra of Compact Riemann Surfaces. Birkhäuser, Boston, 1992.

[9] Cantón, A., Fernández, J. L., Pestana, D. and Rodríguez, J. M., On harmonic functions on trees, Potential Analysis 15 (2001), 199-244.

[10] Chavel, I., Eigenvalues in Riemannian Geometry. Academic Press, New York, 1984.

[11] Coornaert, M., Delzant, T., Papadopoulos, A. Notes sur les groups hyperboliques de Gromov. I.R.M.A., Strasbourg, 1989.

[12] Fenchel, W., Elementary Geometry in Hyperbolic Space. Walter de Gruyter, Berlin-New York, 1989.

[13] Fernández, J. L., Melián, M. V., Escaping geodesics of Riemannian surfaces, Acta Mathematica 187 (2001), 213-236.

[14] Fernández, J. L., Rodríguez, J. M., The exponent of convergence of Riemann surfaces. Bass Riemann surfaces. Ann. Acad. Sci. Fenn. Series AI 15 (1990), 165-183.

[15] Fernández, J. L. and Rodríguez, J. M., Area growth and Green's function of Riemann surfaces, Arkiv för matematik 30 (1992), 83-92.

[16] Ghys, E. and de la Harpe, P., Sur les Groupes Hyperboliques d'après Mikhael Gromov. Progress in Mathematics, Volume 83. Birkhäuser. 1990.

[17] Holopainen, I., Soardi, P. M., p-harmonic functions on graphs and manifolds, Manuscripta Math. 94 (1997), 95-110.

[18] Jones, G. A., Singerman, D., Complex functions. An algebraic and geometric viewpoint. Cambridge Univ. Press, Cambridge, 1987.

[19] Kanai, M., Rough isometries and combinatorial approximations of geometries of non-compact Riemannian manifolds, $J$. Math. Soc. Japan 37 (1985), 391-413.

[20] Kanai, M., Rough isometries and the parabolicity of Riemannian manifolds, J. Math. Soc. Japan 38 (1986), $227-238$.

[21] Nicholls, P. J., The Ergodic Theory of Discrete Groups. Lecture Notes Series 143, Cambridge Univ. Press, Cambridge, 1989.

[22] Paulin, F., On the critical exponent of a discrete group of hyperbolic isometries, Diff. Geom. Appl. 7 (1997), 231-236.

[23] Portilla, A., Rodríguez, J. M., Tourís, E., Gromov hyperbolicity through decomposition of metric spaces II, J. Geom. Anal. 14 (2004), 123-149.

[24] Portilla, A., Rodríguez, J. M., Tourís, E., The topology of balls and Gromov hyperbolicity of Riemann surfaces, Diff. Geom. Appl. 21 (2004), 317-335.

[25] Randol, B., Cylinders in Riemann surfaces, Comment. Math. Helv. 54 (1979), 1-5.

[26] Ratcliffe, J.G., Foundations of Hyperbolic Manifolds. Springer-Verlag, New York, 1994.

[27] Rodríguez, J. M., Tourís, E., Gromov hyperbolicity through decomposition of metric spaces, Acta Math. Hung. 103 (2004), 53-84.

[28] Rodríguez, J. M., Tourís, E., Gromov hyperbolicity of Riemann surfaces. To appear in Acta Math. Sin.

[29] Shimizu, H., On discontinuous groups operating on the product of upper half-planes. Ann. of Math. 77 (1963), 33-71.

[30] Soardi, P. M., Rough isometries and Dirichlet finite harmonic functions on graphs, Proc. Amer. Math. Soc. 119 (1993), $1239-1248$. 
E-mail address: jomaro@math.uc3m.es, etouris@math.uc3m.es 